\title{
Search for top squarks decaying via four-body or chargino-mediated modes in single-lepton final states in proton-proton collisions at $\sqrt{s}=13 \mathrm{TeV}$
}

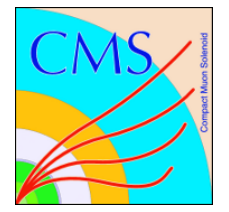

\section{The CMS collaboration}

\section{E-mail: cms-publication-committee-chair@cern.ch}

ABSTRACT: A search for the pair production of the lightest supersymmetric partner of the top quark $\left(\widetilde{t}_{1}\right)$ is presented. The search focuses on a compressed scenario where the mass difference between the top squark and the lightest supersymmetric particle, often considered to be the lightest neutralino $\left(\widetilde{\chi}_{1}^{0}\right)$, is smaller than the mass of the W boson. The proton-proton collision data were recorded by the CMS experiment at a centre-of-mass energy of $13 \mathrm{TeV}$, and correspond to an integrated luminosity of $35.9 \mathrm{fb}^{-1}$. In this search, two decay modes of the top squark are considered: a four-body decay into a bottom quark, two additional fermions, and a $\widetilde{\chi}_{1}^{0}$; and a decay via an intermediate chargino. Events are selected using the presence of a high-momentum jet, significant missing transverse momentum, and a low transverse momentum electron or muon. Two analysis techniques are used, targeting different decay modes of the $\widetilde{t}_{1}$ : a sequential selection and a multivariate technique. No evidence for the production of top squarks is found, and mass limits at $95 \%$ confidence level are set that reach up to $560 \mathrm{GeV}$, depending on the $m\left(\widetilde{\mathrm{t}}_{1}\right)-m\left(\widetilde{\chi}_{1}^{0}\right)$ mass difference and the decay mode.

KEYwords: Hadron-Hadron scattering (experiments), Supersymmetry

ARXIV EPRINT: 1805.05784 


\section{Contents}

1 Introduction $\quad 1$

2 Detector and object definition 3

3 Samples and preselection $\quad 4$

3.1 Data and simulated samples 4

3.2 Preselection 5

4 The CC approach $\quad 6$

4.1 Signal selection 6

4.2 Background prediction 8

4.3 Systematic uncertainties 9

5 The MVA approach $r$

5.1 Signal selection 11

$\begin{array}{lll}5.2 & \text { Background predictions } & 14\end{array}$

$\begin{array}{lll}5.3 & \text { Systematic uncertainties } & 15\end{array}$

6 Results 16

$\begin{array}{lll}7 & \text { Summary } & 18\end{array}$

$\begin{array}{ll}\text { The CMS collaboration } & 28\end{array}$

\section{Introduction}

Searches for new phenomena, in particular supersymmetry (SUSY) [1-6], are among the main objectives of the physics programme at the CERN LHC. Supersymmetry, which is one of the most promising extensions of the standard model (SM), predicts superpartners of SM particles, where the spin of each new particle differs by one-half unit with respect to its SM counterpart. If $R$-parity [7], a new quantum number, is conserved, supersymmetric particles would be pair-produced and their decay chains would end with the lightest supersymmetric particle (LSP). Supersymmetric models can offer solutions to several shortcomings of the SM, in particular those related to the explanation of the mass hierarchy of elementary particles $[8,9]$ and to the presence of dark matter in the universe. The search for SUSY has special interest in view of the recent discovery of the Higgs boson [10-12] as it naturally solves the problem of quadratically divergent loop corrections to the mass of the Higgs boson by associating with each SM particle a supersymmetric partner having the same gauge quantum 

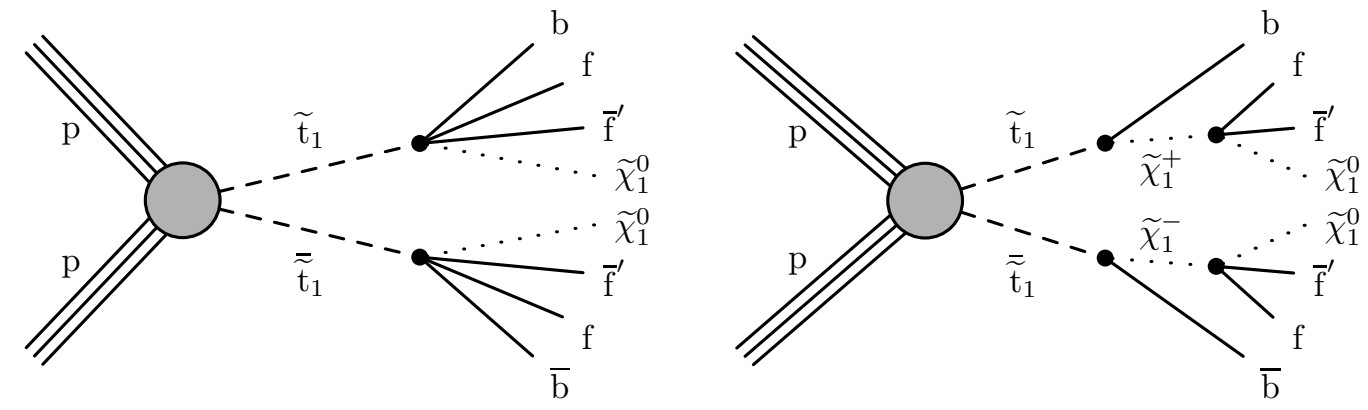

Figure 1. Top squark pair production at the LHC with four-body (left) or chargino-mediated (right) decays.

numbers. In many models of SUSY, the lightest neutralino $\widetilde{\chi}_{1}^{0}$ is the LSP and, being neutral and weakly interacting, would match the characteristics required for a dark matter particle.

Supersymmetry predicts a scalar partner for each SM left- and right-handed fermion. When SUSY is broken, the scalar partners acquire masses different from those of their SM counterparts, and the mass splitting between the two squark mass eigenstates is proportional to the mass of their SM partner. Given the large mass of the top quark, this splitting can be the largest among all squarks. Therefore the lightest supersymmetric partner of the top quark, the $\widetilde{t}_{1}$, is often the lightest squark. Furthermore, if SUSY is a symmetry of nature, cosmological observations may suggest the lightest top squark to be almost degenerate with the LSP [13]. This motivates the search for a four-body $\widetilde{t}_{1}$ decay: $\widetilde{\mathrm{t}}_{1} \rightarrow \mathrm{bf} \overline{\mathrm{f}}^{\prime} \widetilde{\chi}_{1}^{0}$, where the fermions $\mathrm{f}$ and $\overline{\mathrm{f}}^{\prime}$ can be either quarks or leptons. Here, due to the small mass difference between the $\widetilde{\mathrm{t}}_{1}$ and the $\widetilde{\chi}_{1}^{0}$, two-body $\left(\widetilde{\mathrm{t}}_{1} \rightarrow \mathrm{t} \widetilde{\chi}_{1}^{0}, \widetilde{\mathrm{t}}_{1} \rightarrow \mathrm{b} \tilde{\chi}_{1}^{+}\right)$and three-body $\left(\widetilde{\mathrm{t}}_{1} \rightarrow \mathrm{bW}^{+} \widetilde{\chi}_{1}^{0}\right)$ decays of the lightest top squark are kinematically forbidden, and the two-body $\left(\widetilde{\mathrm{t}}_{1} \rightarrow \mathrm{c} \widetilde{\chi}_{1}^{0}\right)$ decay can be suppressed depending on the details of the model. Alternatively, the decay $\widetilde{t}_{1} \rightarrow \mathrm{b} \tilde{\chi}_{1}^{+} \rightarrow \mathrm{bf} \bar{f}^{\prime} \widetilde{\chi}_{1}^{0}$ is possible if the mass of the lightest chargino is lower than the top squark mass. Figure 1 represents the production of a pair of $\widetilde{t}_{1}$ followed by a four-body or chargino-mediated decay in simplified models [14].

In this paper, we describe a search for pair production of the $\widetilde{t}_{1}$ in proton-proton (pp) collisions at the LHC at $\sqrt{s}=13 \mathrm{TeV}$, where each top squark can decay either directly, or via a chargino, into the $\mathrm{bf}^{\prime} \widetilde{\chi}_{1}^{0}$ final state. A $100 \%$ branching fraction for each decay is assumed when interpreting the results [14]. The final states considered contain jets, missing transverse momentum ( $\left.p_{\mathrm{T}}^{\text {miss }}\right)$, and exactly one lepton, which can be either an electron or a muon, originating from the decay of the top squark or the chargino, depending on the considered decay scenario. The lepton can be efficiently reconstructed and identified with transverse momentum $\left(p_{\mathrm{T}}\right)$ as low as 5.0 and $3.5 \mathrm{GeV}$ for electrons and muons, respectively. In this search, we expand the result of a previous CMS search in pp collisions at $\sqrt{s}=8 \mathrm{TeV}[15]$ by including the single-electron final state and lowering the $p_{\mathrm{T}}$ thresholds for leptons. Moreover, two different approaches are used in this analysis. A signal selection based on sequentially applied requirements on several discriminating variables (CC) has been designed to provide good sensitivity over a wide range of kinematic signatures corre- 
sponding to different $\left(m\left(\widetilde{\mathrm{t}}_{1}\right), m\left(\widetilde{\chi}_{1}^{0}\right)\right)$ mass hypotheses and different $\widetilde{\mathrm{t}}_{1}$ decay modes. The $\mathrm{CC}$ approach is applied to the four-body and chargino-mediated $\widetilde{t}_{1}$ decay scenarios. In addition, a multivariate analysis (MVA) followed by a counting experiment approach is used for the signal selection. Applied to the four-body scenario, this approach exploits the correlations between discriminating variables and is adapted for different $\Delta m=m\left(\widetilde{\mathrm{t}}_{1}\right)-m\left(\widetilde{\chi}_{1}^{0}\right)$ kinematic regions, thus optimizing the search across the $\left(m\left(\widetilde{\mathrm{t}}_{1}\right), m\left(\widetilde{\chi}_{1}^{0}\right)\right)$ space and improving upon the sensitivity of the CC approach for this scenario. Both approaches are based on a nearly identical preselection.

Other results in the single-lepton final state and for both the four-body and charginomediated $\widetilde{\mathrm{t}}_{1}$ decays were reported by ATLAS at $\sqrt{s}=8 \mathrm{TeV}[16]$ and $\sqrt{s}=13 \mathrm{TeV}$ [17]. Other final states at $\sqrt{s}=13 \mathrm{TeV}$ were investigated by ATLAS and CMS for all-hadronic events $[18,19]$ and for final states with two isolated leptons $[20,21]$, respectively.

\section{Detector and object definition}

The central feature of the CMS apparatus is a superconducting solenoid of $6 \mathrm{~m}$ internal diameter, providing a magnetic field of $3.8 \mathrm{~T}$. Within the solenoid volume are a silicon pixel and strip tracker, a lead tungstate crystal electromagnetic calorimeter (ECAL), and a brass and scintillator hadron calorimeter (HCAL), each composed of a barrel and two endcap sections. Forward calorimeters extend the pseudorapidity coverage provided by the barrel and endcap detectors. The silicon tracker measures charged particles within the pseudorapidity range $|\eta|<2.5$. Muons are detected in gas-ionization chambers embedded in the steel flux-return yoke outside the solenoid. The detector is nearly hermetic, allowing for momentum balance measurements in the plane transverse to the beam axis. Events are selected for further analysis by a two-tier trigger system that uses custom hardware processors to make a fast initial selection, followed by a more detailed selection executed on a dedicated processor farm. A more detailed description of the CMS detector can be found in ref. [22].

This analysis utilizes the CMS particle-flow (PF) algorithm [23] to reconstruct and identify PF candidates such as leptons (electrons and muons), photons, and charged and neutral hadrons. The reconstructed vertex with the largest value of summed physics-object $p_{T}^{2}$ is taken to be the primary pp interaction vertex $(\mathrm{PV})$. The physics objects are the jets, clustered using a jet finding algorithm [24, 25] with the tracks assigned to the vertex as inputs, and the associated missing transverse momentum, taken as the negative vector sum of the $p_{\mathrm{T}}$ of those jets.

The electron candidates are reconstructed from energy depositions in the ECAL and from tracks in the inner tracker obtained using the Gaussian-sum filter [26]. The misidentification of electrons is reduced by requiring additional constraints on the shape of the electromagnetic shower in the ECAL, the quality of the match between the trajectory of the track and the ECAL energy deposit, and the relative HCAL deposition in the electron direction. For reconstructing muons the tracks in both the silicon tracker and the muon system are used [27]. The number of measurements in the tracker and muon system and the quality of the track fit are used to reduce the misidentification rate of muons. In order to select leptons $(\ell=\mathrm{e}$ or $\mu$ ) from the primary interaction, the point of closest approach 
to the PV of tracks associated with the lepton is required to have transverse component $\left|d_{x y}\right|<0.02 \mathrm{~cm}$ and longitudinal component $\left|d_{z}\right|<0.1 \mathrm{~cm}$ with respect to the PV. In order to suppress the selection of nonprompt leptons, which may arise from jets produced in association with the invisible decay of a $\mathrm{Z}$ boson, multijet production, or $\mathrm{W}+$ jets and $\mathrm{t} \overline{\mathrm{t}}$ events with a lost lepton, selected leptons are required to be isolated from jet activity by using a combination of absolute and relative isolation variables. The absolute isolation $\left(I_{\text {abs }}\right)$ of the lepton is defined as the scalar sum of the $p_{\mathrm{T}}$ of PF candidates within a cone size of $R \equiv \sqrt{(\Delta \phi)^{2}+(\Delta \eta)^{2}}=0.3$. The leptons and charged PF candidates not associated with the PV are not included in the sum. The contribution of the neutral particles from simultaneous pp collisions (pileup) is estimated according to the method described in ref. [26], and subtracted from $I_{\text {abs }}$. The relative isolation $\left(I_{\text {rel }}\right)$ of a lepton is defined as the ratio of lepton $I_{\mathrm{abs}}$ to the lepton $p_{\mathrm{T}}$. The electrons and muons are then required to satisfy $I_{\text {abs }}<5 \mathrm{GeV}$ for $p_{\mathrm{T}}(\ell)<25 \mathrm{GeV}$ and $I_{\text {rel }}<0.2$ for $p_{\mathrm{T}}(\ell)>25 \mathrm{GeV}$. This combined isolation criterion allows for a more uniform selection efficiency of leptons as a function of lepton $p_{\mathrm{T}}$. Finally, the selected electrons and muons are also required to have $p_{\mathrm{T}}$ above $5.0 \mathrm{GeV}$ and $3.5 \mathrm{GeV}$ and $|\eta|<2.5$ and 2.4 , respectively. Tau leptons with a hadronic decay are reconstructed from the PF candidates using the "hadrons-plus-strips" algorithm [28], which achieves an efficiency of 50-60\%. The tau candidates are required to satisfy $|\eta|<2.4$.

The jets used in this analysis are reconstructed by clustering PF candidates using the anti- $k_{\mathrm{T}}$ algorithm [24] with a distance parameter of 0.4. The missing transverse momentum vector, $\vec{p}_{\mathrm{T}}^{\text {miss }}$, in the event is defined as the negative of the vectorial sum of the transverse momenta of all the PF candidates in the event with its magnitude denoted as $p_{\mathrm{T}}^{\text {miss }}$. The pileup contribution to the jet momenta is partially taken into account by subtracting the energy of charged hadrons originating from a vertex other than the PV. The jet momenta are further calibrated to account for contributions from neutral pileup and any inhomogeneities of detector response [29]. The jets have a threshold of $p_{\mathrm{T}}>30 \mathrm{GeV}$ and are required to have $|\eta|<2.4$.

Jets originating from bottom (b) quarks are identified ("tagged") as "b jets" using the combined secondary vertex algorithm [30,31], which takes advantage of MVA techniques. The medium working point of this algorithm is used in the CC search, which has a probability of about $1 \%$ to misidentify a light quark jet as a $b$ jet while correctly identifying a $\mathrm{b}$ jet with an efficiency of about $65 \%$. The same figures for the loose working point, which is used in the MVA search, are $10 \%$ and $80 \%$, respectively.

\section{Samples and preselection}

\subsection{Data and simulated samples}

The searches described in this paper are performed using data from pp collisions recorded in 2016 by the CMS experiment at the LHC at a centre-of-mass energy of $13 \mathrm{TeV}$ corresponding to an integrated luminosity of $35.9 \mathrm{fb}^{-1}$. Events in the search are collected based on $p_{\mathrm{T}}^{\text {miss }}$ triggers with thresholds ranging between 90 and $120 \mathrm{GeV}$. Additional control samples used for estimating backgrounds are selected by single-lepton triggers with $p_{\mathrm{T}}$ thresholds of 24 and $27 \mathrm{GeV}$ for muons and electrons, respectively. 
In this analysis, Monte Carlo (MC) simulation samples of SM processes are used to relate background yields in control and signal regions, to validate the background estimation methods based on data, and to predict contributions from rare processes. Simulated samples are produced using multiple generators. The main background samples, namely $\mathrm{W}+$ jets, $\mathrm{t} \overline{\mathrm{t}}$, and $\mathrm{Z} / \gamma^{*}$ are generated at leading order (LO) by MADGRAPH5_aMC@NLO 2.3.3 [32]. Next-to-leading order (NLO) simulations with the POWHEG v2.0 [33] and POWHEG v1.0 [34] generators are used for single top quark production and the associated tW production, respectively. Diboson events are simulated at NLO with MadGraph5_amC@NLO 2.3.3 and POWHEG v2.0. The LO and NLO NNPDF3.0 [35] parton distribution functions (PDFs) are used consistently with the order of the matrix element calculation in the generated events. Hadronization and showering of events in all generated samples have been simulated using PYTHIA 8.212 [36, 37] with the CUETP8M1 [38] tune for the underlying event. The response of the CMS detector is modelled using the GEANT4 [39] program. Simulation and data events are reconstructed with the same algorithms. The effect of pileup is simulated in the MC samples in order to reproduce the observed pileup conditions in data.

The signal samples for the pair production of top squarks $\left(\widetilde{\mathrm{t}}_{1} \overline{\mathrm{t}}_{1}\right)$ are simulated for $250 \leq m\left(\widetilde{\mathrm{t}}_{1}\right) \leq 800 \mathrm{GeV}$ in steps of $25 \mathrm{GeV}$, and $10 \leq \Delta m \leq 80 \mathrm{GeV}$ in $10 \mathrm{GeV}$ steps. The cross section for $\widetilde{\mathrm{t}}_{1} \overline{\widetilde{\mathrm{t}}}_{1}$ production at NLO and including next-to-leading logarithmic (NLL) corrections, as calculated by PROSPINO v.2 [40-46], varies approximately between 20 and 0.1 $\mathrm{pb}$ for the mass range considered. The pair production of squarks with up to two additional jets from initial-state radiation (ISR) is generated with MADGRAPH5_aMC@NLO 2.3.3 and is then interfaced with PYTHIA 8.212 for the decay, hadronization, and showering. For the chargino-mediated decay of the scalar top, $m\left(\widetilde{\chi}_{1}^{ \pm}\right)$is taken to be the average of $m\left(\widetilde{t}_{1}\right)$ and $m\left(\widetilde{\chi}_{1}^{0}\right)$. The decay is generated to proceed via an off-shell $\mathrm{W}$ boson, and the $\widetilde{\mathrm{t}}_{1}$ decay length is set to zero. The modelling of the detector response is performed with the CMS fast simulation program [47].

Simulated background and signal samples are corrected for differences with respect to the values measured in data control samples in the selection efficiencies for leptons and $b$ jets, and for the misidentification probability for light-quark and gluon jets as $\mathrm{b}$ jets. These corrections are applied as functions of the $p_{\mathrm{T}}$ and $\eta$ of the objects. For the signal samples, additional corrections are applied to take into account any potential differences between the GEANT4 and fast simulations in regards to tagging efficiencies of b jets, leptons, and modelling of $p_{\mathrm{T}}^{\mathrm{miss}}$.

\subsection{Preselection}

The preselection requirements used in this paper are designed by considering the general characteristics of the signal, and are based on the methods presented in ref. [15]. The CC and MVA approaches share similar preselection requirements with a few minor differences that are noted below due to studies showing that the MVA leads to better performance with slightly different selection than the $\mathrm{CC}$ search. In order to match the trigger requirement, events with $p_{\mathrm{T}}^{\text {miss }}>200(280) \mathrm{GeV}$ are selected for the CC (MVA) approach. This requirement favours the signal, which tends to have larger missing transverse momentum 
than SM processes due to two $\widetilde{\chi}_{1}^{0}$ 's escaping detection. The efficiency of signal triggers is measured to be higher than $90(98) \%$ for $p_{\mathrm{T}}^{\text {miss }}>200(280) \mathrm{GeV}$, and the simulated samples are reweighted as a function of $p_{\mathrm{T}}^{\text {miss }}$ to account for the inefficiency.

Further suppression of SM processes such as $\mathrm{W}+$ jets is achieved by imposing the additional requirement of $H_{\mathrm{T}}>300 \mathrm{GeV}$, where $H_{\mathrm{T}}$ is defined as the scalar $p_{\mathrm{T}}$ sum of all jets. For the MVA search, this requirement is relaxed to $H_{\mathrm{T}}>200 \mathrm{GeV}$. In order to improve the separation of signal and SM background, we take advantage of events in which the $\widetilde{t}_{1}$ pair system recoils against an ISR jet. In this case the LSP becomes Lorentz boosted, which increases the $p_{\mathrm{T}}^{\text {miss }}$ in the event, while jets and leptons remain relatively soft. The ISR jet candidate in the event is selected as the leading jet with $|\eta|<2.4$, which is required to satisfy $p_{\mathrm{T}}>100$ (110) GeV for the CC (MVA) search. To reduce the contribution from $\mathrm{t} \overline{\mathrm{t}}$ production, events are required to have at most two jets with $p_{\mathrm{T}}>60 \mathrm{GeV}$ in the CC search. In events with two jets, the azimuthal angle between the leading and subleading (in $p_{\mathrm{T}}$ ) jets is required to be less than 2.5 radians in order to suppress the SM multijet background.

Finally, the soft single-lepton topology is selected by requiring at least one muon or electron in the event, while vetoing events with a $\tau$ lepton, or a second electron or muon with $p_{\mathrm{T}}>20 \mathrm{GeV}$. At this stage of the selection, the $\mathrm{W}+\mathrm{jets}$ and $\mathrm{t} \overline{\mathrm{t}}$ processes represent approximately $70 \%$ and $20 \%$ of the total expected background, respectively. The $\mathrm{Z}(\rightarrow \nu \nu)+$ jets process contributes with jets, genuine $p_{\mathrm{T}}^{\text {miss }}$, and a jet misidentified as a lepton. Diboson, single top and Drell-Yan (DY) processes also contribute, with a lower expected yield due to a low cross section or a low acceptance (or both).

\section{The CC approach}

\subsection{Signal selection}

After the preselection detailed in the previous section, $\mathrm{W}+$ jets is the dominant background process, followed by a smaller contribution coming from t $\bar{t}$ production. A kinematic variable with good discrimination against these background processes is the transverse mass $M_{\mathrm{T}} \equiv$ $\sqrt{2 p_{\mathrm{T}}^{\text {miss }} p_{\mathrm{T}}(\ell)(1-\cos \Delta \phi)}$, where $p_{\mathrm{T}}(\ell)$ is the transverse momentum of the selected lepton and $\Delta \phi$ is the angular difference between the lepton $\vec{p}_{\mathrm{T}}(\ell)$ and $\vec{p}_{\mathrm{T}}^{\text {miss }}$. The distributions of lepton $p_{\mathrm{T}}$ and $M_{\mathrm{T}}$ are shown in figure 2 for the observed data and simulated background and signal, where we observe good agreement in the shapes of the distributions between data and background simulation. The normalization of the simulation is corrected by the results of a background estimation technique based partially on data, as described in section 4.2.

The signal regions (SRs) in the CC analysis are defined to maximize the sensitivity of the search by exploiting the differences between the kinematic properties of the final-state particles in the signal and background processes. The leptons originating from the decay of the $\widetilde{t}_{1}$ squark are expected to be much softer than those from SM processes. Therefore, all SRs are required to satisfy $p_{\mathrm{T}}(\ell)<30 \mathrm{GeV}$. In order to retain sensitivity to different $\Delta m$ mass gaps, two signal regions SR1 and SR2 are designed targeting small and large mass differences, respectively. Moreover, due to the strong dependence of the $p_{\mathrm{T}}$ and $M_{\mathrm{T}}$ distributions for the signal on $\Delta m$, these SRs are further subdivided into a total of 44 mutually exclusive regions, which are detailed below and summarized in table 1 . In 

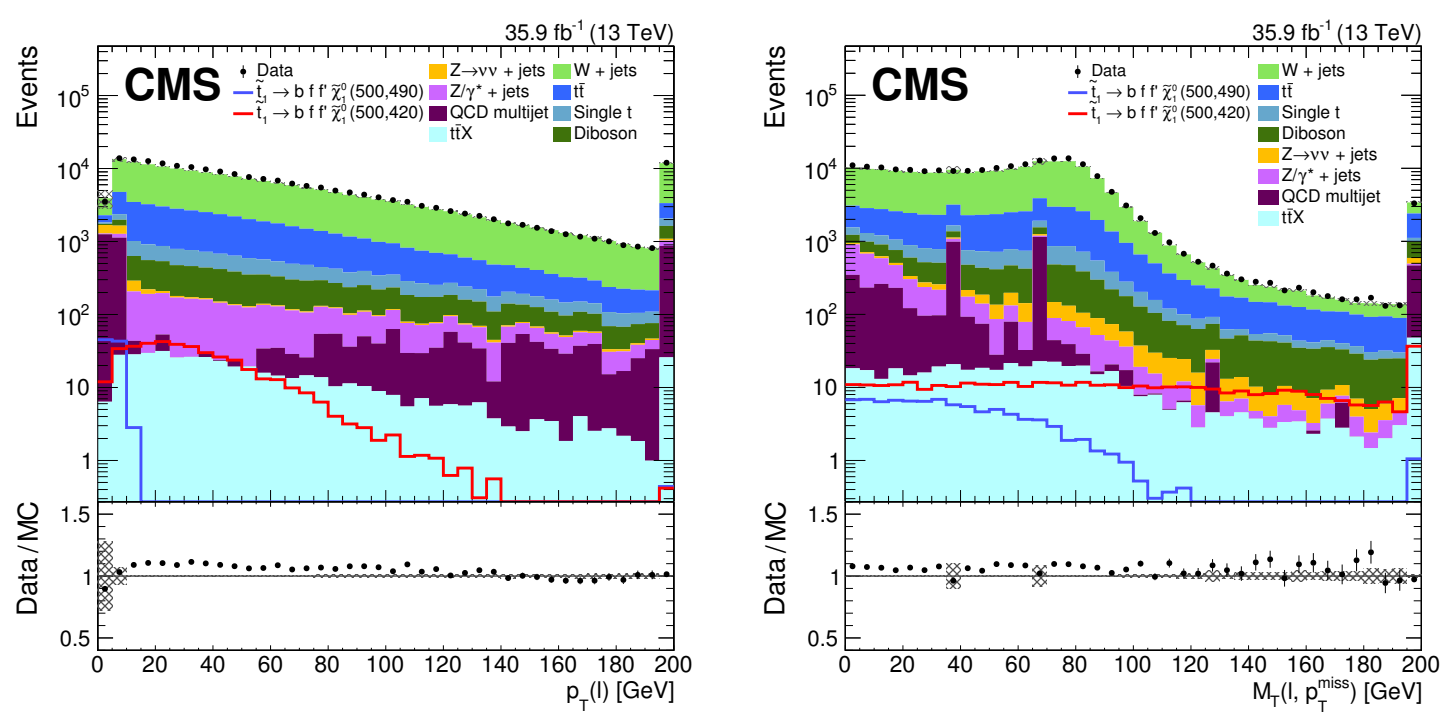

Figure 2. Distributions of lepton $p_{\mathrm{T}}$ (left) and $M_{\mathrm{T}}$ (right) at the preselection level in data and simulation. The lower panels show the ratio of data to the sum of the SM backgrounds where the dark shaded bands indicate the statistical uncertainties of the simulation. The distributions of two signal points of the four-body decay are also represented, while not being added to the background: $\left(m\left(\widetilde{\mathrm{t}}_{1}\right), m\left(\widetilde{\chi}_{1}^{0}\right)\right)=(500,490)$ and $(500,420) \mathrm{GeV}$. The background distributions are obtained directly from simulation, and are normalized to an integrated luminosity of $35.9 \mathrm{fb}^{-1}$. The last bin in each plot includes events beyond $200 \mathrm{GeV}$.

this search, the correlations between $p_{\mathrm{T}}^{\text {miss }}, H_{\mathrm{T}}$, and the transverse momentum of the ISR jet candidate $\left(p_{\mathrm{T}}(\mathrm{ISR})\right)$ are taken into account by defining SRs in terms of the variables $C_{\mathrm{T} 1}$ and $C_{\mathrm{T} 2}: C_{\mathrm{T} 1} \equiv \min \left(p_{\mathrm{T}}^{\text {miss }}, H_{\mathrm{T}}-100 \mathrm{GeV}\right)$, and $C_{\mathrm{T} 2} \equiv \min \left(p_{\mathrm{T}}^{\text {miss }}, p_{\mathrm{T}}(\mathrm{ISR})-25 \mathrm{GeV}\right)$, where the numerical values of 100 and $25 \mathrm{GeV}$ are determined by maximizing the ratio of signal to the square root of background in the signal regions.

In SR1, events with a b jet satisfying $p_{\mathrm{T}}>30 \mathrm{GeV}$ are rejected since the b jets in signal events with a small mass gap are expected to have typical $p_{\mathrm{T}}$ values smaller than this threshold. This b-tag veto significantly reduces the contribution of $t \bar{t}$ events. In this region, the $p_{\mathrm{T}}^{\text {miss }}$ and $H_{\mathrm{T}}$ requirements of the preselection are simultaneously tightened by requiring $C_{\mathrm{T} 1}>300 \mathrm{GeV}$. Since the $\mathrm{W}+$ jets process is the dominant background process for lower $M_{\mathrm{T}}$ values, we take advantage of the charge asymmetry in the production of $\mathrm{W}$ bosons at LHC and require the lepton to have a negative charge in SR1 regions with $M_{\mathrm{T}}<95 \mathrm{GeV}$. Moreover, the acceptance of the lepton is tightened by requiring $|\eta(\ell)|<1.5$, because leptons from decays of the $\mathrm{W}$ boson at the LHC tend to be produced in the forward direction.

In SR2, we require at least one b jet with $p_{\mathrm{T}}<60 \mathrm{GeV}$, but reject events with any b jet having $p_{\mathrm{T}}>60 \mathrm{GeV}$. These requirements increase the efficiency of signal points with larger $\Delta m$ while keeping the $t \bar{t}$ background under control. In this region we also require $C_{\mathrm{T} 2}>300 \mathrm{GeV}$, which is more effective in reducing the $\mathrm{t} \overline{\mathrm{t}}$ background compared to the $C_{\mathrm{T} 1}$ requirement.

The SR1 (SR2) region is further divided in bins of $M_{\mathrm{T}}$, lepton $p_{\mathrm{T}}$, and $C_{\mathrm{T} 1}\left(C_{\mathrm{T} 2}\right)$. The $M_{\mathrm{T}}$ binning is done below and above the peak around the $\mathrm{W}$ boson mass in the 


\begin{tabular}{|c|c|c|c|c|c|c|}
\hline Variable & \multicolumn{6}{|c|}{ Common to all SRs } \\
\hline Number of hard jets & \multicolumn{6}{|c|}{$\leq 2$} \\
\hline$\Delta \phi($ hard jets $)(\mathrm{rad})$ & \multicolumn{6}{|c|}{$<2.5$} \\
\hline$p_{\mathrm{T}}^{\text {miss }}(\mathrm{GeV})$ & \multicolumn{6}{|c|}{$>300$} \\
\hline Lepton rejection & \multicolumn{6}{|c|}{ no $\tau$, or additional $\ell$ with $p_{\mathrm{T}}>20 \mathrm{GeV}$} \\
\hline & \multicolumn{3}{|c|}{ SR1 } & \multicolumn{3}{|c|}{ SR2 } \\
\hline$H_{\mathrm{T}}(\mathrm{GeV})$ & \multicolumn{3}{|c|}{$>400$} & \multicolumn{3}{|c|}{$>300$} \\
\hline$p_{\mathrm{T}}(\mathrm{ISR}$ jet $)(\mathrm{GeV})$ & \multicolumn{3}{|c|}{$>100$} & \multicolumn{3}{|c|}{$>325$} \\
\hline Number of b jets & \multicolumn{3}{|c|}{0} & \multicolumn{3}{|c|}{$\geq 1$ soft, 0 hard } \\
\hline$|\eta(\ell)|$ & \multicolumn{3}{|c|}{$<1.5$} & \multicolumn{3}{|c|}{$<2.4$} \\
\hline & SR1a & SR1b & SR1c & SR2a & SR2b & SR2c \\
\hline$M_{\mathrm{T}}(\mathrm{GeV})$ & $<60$ & $60-95$ & $>95$ & $<60$ & $60-95$ & $>95$ \\
\hline$Q(\ell)$ & -1 & -1 & any & any & any & any \\
\hline$p_{\mathrm{T}}(\mu)(\mathrm{GeV})$ & $3.5-5(\mathrm{VL})$ & $3.5-5(\mathrm{VL})$ & - & $3.5-5(\mathrm{VL})$ & $3.5-5(\mathrm{VL})$ & - \\
\hline \multirow[t]{4}{*}{$p_{\mathrm{T}}(\mathrm{e}, \mu)(\mathrm{GeV})$} & $5-12(\mathrm{~L})$ & $5-12(\mathrm{~L})$ & 5-12(L) & $5-12(\mathrm{~L})$ & 5-12(L) & 5-12(L) \\
\hline & $12-20(\mathrm{M})$ & $12-20(\mathrm{M})$ & $12-20(\mathrm{M})$ & $12-20(\mathrm{M})$ & $12-20(\mathrm{M})$ & $12-20(\mathrm{M})$ \\
\hline & $20-30(\mathrm{H})$ & $20-30(\mathrm{H})$ & $20-30(\mathrm{H})$ & $20-30(\mathrm{H})$ & $20-30(\mathrm{H})$ & $20-30(\mathrm{H})$ \\
\hline & $>30(\mathrm{CR})$ & $>30(\mathrm{CR})$ & $>30(\mathrm{CR})$ & $>30(\mathrm{CR})$ & $>30(\mathrm{CR})$ & $>30(\mathrm{CR})$ \\
\hline$C_{\mathrm{T}}(\mathrm{GeV})$ & \multicolumn{3}{|c|}{$\begin{array}{c}300<C_{\mathrm{T} 1}<400(\mathrm{X}) \\
C_{\mathrm{T} 1}>400(\mathrm{Y})\end{array}$} & \multicolumn{3}{|c|}{$\begin{array}{c}300<C_{\mathrm{T} 2}<400(\mathrm{X}) \\
C_{\mathrm{T} 2}>400(\mathrm{Y})\end{array}$} \\
\hline
\end{tabular}

Table 1. The CC search: definition of SRs. The subregions of SRs are denoted by tags in parentheses, as described in the text: $\mathrm{VL}, \mathrm{L}, \mathrm{M}$, and $\mathrm{H}$ refer to the four bins in lepton $p_{\mathrm{T}}$, and $\mathrm{X}$ and $\mathrm{Y}$ to the $C_{\mathrm{T}}$ ranges specified in the table. The corresponding control regions (CR) use the same selection with the exception of the lepton $p_{\mathrm{T}}$ as shown in the table. For jets, the attributes "soft" and "hard" refer to the $p_{\mathrm{T}}$ ranges $30-60 \mathrm{GeV}$ and $>60 \mathrm{GeV}$, respectively.

$M_{\mathrm{T}}$ distribution, with the regions $M_{\mathrm{T}}<60 \mathrm{GeV}, 60<M_{\mathrm{T}}<95 \mathrm{GeV}$, and $M_{\mathrm{T}}>95 \mathrm{GeV}$ labelled as a, b, and c, respectively. It can be seen from figure 2 that the lower (higher) $M_{\mathrm{T}}$ region is more sensitive to signals with smaller (larger) mass gaps. In order to take advantage of the shape differences in lepton $p_{\mathrm{T}}$ distributions between various signal points and SM processes, each SR is further divided into lepton $p_{\mathrm{T}}$ regions $5-12,12-20$, and 20 $30 \mathrm{GeV}$, referred to as $\mathrm{L}, \mathrm{M}$, and $\mathrm{H}$, respectively. An additional region of $3.5-5.0 \mathrm{GeV}$ is added only for muons and only for $M_{\mathrm{T}}<95 \mathrm{GeV}$, and is labelled VL. In addition, SR1 (SR2) is further separated into two regions in $C_{\mathrm{T} 1}\left(C_{\mathrm{T} 2}\right)$ defined by $300<C_{\mathrm{T} 1}\left(C_{\mathrm{T} 2}\right)<400 \mathrm{GeV}$ and $C_{\mathrm{T} 1}\left(C_{\mathrm{T} 2}\right)>400 \mathrm{GeV}$ which are labelled $\mathrm{X}$ and $\mathrm{Y}$, respectively.

\subsection{Background prediction}

The dominant backgrounds in most of the $\mathrm{CC}$ signal regions are $\mathrm{W}+$ jets and $\mathrm{t} \overline{\mathrm{t}}$ production with a prompt lepton in the final state. The nonprompt sources of leptons become more important in regions with large $M_{\mathrm{T}}$ or very low lepton $p_{\mathrm{T}}$. In this section, the methods used 
to estimate the prompt and nonprompt backgrounds from data are described. Simulation is used to estimate other rare backgrounds with a prompt lepton, namely $\mathrm{Z} / \gamma^{*}$, diboson, single top quark production, and $\mathrm{t} \overline{\mathrm{t}}$ production with an additional $\mathrm{W}, \mathrm{Z}$, or $\gamma$.

The nonprompt background due to misidentified leptons associated with a jet becomes comparable to the prompt contribution in regions where $\mathrm{W}+$ jets and $\mathrm{t} \overline{\mathrm{t}}$ production are suppressed, namely in regions of high- $M_{\mathrm{T}}$ and very low lepton $p_{\mathrm{T}}$. This background is estimated fully from data using the "tight-to-loose" method, where a "loose" set of identification and isolation criteria are defined to select lepton candidates that are more likely to be nonprompt. The loose selection is defined by relaxing the requirement on the lepton isolation to $I_{\mathrm{abs}}<20 \mathrm{GeV}$ for $p_{\mathrm{T}}(\ell)<25 \mathrm{GeV}$ and $I_{\text {rel }}<0.8$ for $p_{\mathrm{T}}(\ell)>25 \mathrm{GeV}$, as well as relaxing the impact parameter conditions to $\left|d_{x y}\right|<0.1 \mathrm{~cm}$ and $\left|d_{z}\right|<0.5 \mathrm{~cm}$. The "tight" criteria correspond to the final lepton selection of the analysis, described in section 2 . The probability that a loose lepton also passes the tight criteria, the tight-to-loose fraction $\epsilon_{\mathrm{TL}}$, is measured as a function of lepton $p_{\mathrm{T}}$ and $|\eta|$ in an orthogonal "measurement region" largely dominated by multijet events, which is enriched in nonprompt leptons. The fraction $\epsilon_{\mathrm{TL}}$ is measured from data, after the subtraction of the simulated prompt lepton contribution. The final estimate of nonprompt leptons in a SR or control region (CR) is based on the observed yield in an "application region". The latter is defined in the same way as the corresponding SR or CR, with the exception that the lepton has to pass the loose lepton criteria but not the tight ones. The final estimate is obtained by scaling the data yield in the application region by $\epsilon_{\mathrm{TL}} /\left(1-\epsilon_{\mathrm{TL}}\right)$, after subtracting the simulated prompt lepton contribution.

The absolute normalization of the prompt background simulation in each SR is obtained from a $\mathrm{CR}$ with identical requirements as in the SR except for the lepton $p_{\mathrm{T}}$ selection. The $\mathrm{CR}$ is defined by replacing the lepton $p_{\mathrm{T}}$ requirement of the $\mathrm{SR}$ with $p_{\mathrm{T}}(\ell)>30 \mathrm{GeV}$; therefore, SRs that are only distinguished by different selections in $p_{\mathrm{T}}(\ell)$ share the same CR. The impact of potential signal contamination is taken into account when deriving the results as described in section 6 . In each $\mathrm{CR}$, a scale factor for the prompt simulation is obtained by normalizing the simulation to data, after subtracting nonprompt and rare background sources from the observed number of events in the CR. The nonprompt contribution used in the subtraction is estimated separately from data. The composition of the CRs in terms of background processes, as well as the total simulated and observed yields, are shown in table 2. The scale factors, ranging from 0.86 to 1.25 , are then applied to the simulation in the corresponding SRs. In order to verify the extrapolation of the scale factors from CR to SR, we perform the same background estimation procedure in validation regions (VRs), which are orthogonal to all SRs and CRs. Each validation region is obtained by one of the following changes: (a) lowering the $C_{\mathrm{T} 1}$ (in SR1 and CR1) and $C_{\mathrm{T} 2}$ (in SR2 and CR2) requirements to $200<C_{\mathrm{T}}<300 \mathrm{GeV}$, (b) replacing the conditions on $\mathrm{b}$ jets by requiring at least one $\mathrm{b}$ jet with $p_{\mathrm{T}}>60 \mathrm{GeV}$. The predictions in the validation regions are compatible with the observations within the uncertainties.

\subsection{Systematic uncertainties}

Processes for which the absolute yield is predicted by simulation are subject to systematic uncertainties in the determination of the integrated luminosity (2.5\%) [48]. All simulated 


\begin{tabular}{|lccccccc|}
\hline Region & W+jets & $\mathrm{t} \overline{\mathrm{t}}$ & Nonprompt & Rare & Total SM & Data & Scale factors \\
\hline $\mathrm{CR} 1 \mathrm{aX}$ & $2133 \pm 20$ & $226.6 \pm 3.5$ & $44.5 \pm 6.4$ & $293.2 \pm 5.9$ & $2698 \pm 22$ & 2945 & $1.10 \pm 0.03$ \\
$\mathrm{CR} 1 \mathrm{aY}$ & $878.3 \pm 8.6$ & $65.8 \pm 1.9$ & $13.3 \pm 3.6$ & $139.4 \pm 4.1$ & $1097 \pm 10$ & 1197 & $1.11 \pm 0.04$ \\
$\mathrm{CR} 1 \mathrm{bX}$ & $1107 \pm 15$ & $134.5 \pm 2.7$ & $7.8 \pm 2.7$ & $112.1 \pm 4.1$ & $1361 \pm 16$ & 1462 & $1.08 \pm 0.03$ \\
$\mathrm{CR} 1 \mathrm{bY}$ & $438.2 \pm 6.4$ & $35.1 \pm 1.4$ & $1.6 \pm 1.6$ & $51.9 \pm 2.9$ & $526.8 \pm 7.3$ & 502 & $0.95 \pm 0.05$ \\
$\mathrm{CR} 1 \mathrm{cX}$ & $642 \pm 11$ & $103.8 \pm 2.3$ & $12.7 \pm 3.0$ & $174.3 \pm 5.5$ & $932 \pm 13$ & 1051 & $1.16 \pm 0.05$ \\
$\mathrm{CR} 1 \mathrm{cY}$ & $278.3 \pm 8.3$ & $25.5 \pm 1.2$ & $6.2 \pm 2.2$ & $102.2 \pm 4.3$ & $412.2 \pm 9.6$ & 432 & $1.07 \pm 0.08$ \\
$\mathrm{CR} 2 \mathrm{aX}$ & $171.7 \pm 2.5$ & $195.6 \pm 3.3$ & $1.9 \pm 1.9$ & $64.2 \pm 1.9$ & $433.4 \pm 4.9$ & 451 & $1.05 \pm 0.06$ \\
$\mathrm{CR} 2 \mathrm{aY}$ & $74.5 \pm 1.0$ & $58.4 \pm 1.7$ & $0.8 \pm 0.8$ & $25.6 \pm 1.1$ & $159.3 \pm 2.4$ & 145 & $0.89 \pm 0.09$ \\
$\mathrm{CR} 2 \mathrm{bX}$ & $104.9 \pm 2.0$ & $110.8 \pm 2.5$ & $1.2 \pm 1.2$ & $39.2 \pm 1.6$ & $256.1 \pm 3.8$ & 226 & $0.86 \pm 0.07$ \\
$\mathrm{CR} 2 \mathrm{bY}$ & $42.6 \pm 0.8$ & $30.8 \pm 1.3$ & $0.3 \pm 0.3$ & $15.0 \pm 0.9$ & $88.6 \pm 1.8$ & 79 & $0.87 \pm 0.12$ \\
$\mathrm{CR} 2 \mathrm{cX}$ & $17.3 \pm 0.8$ & $53.8 \pm 1.7$ & $1.7 \pm 1.2$ & $15.7 \pm 1.0$ & $88.4 \pm 2.4$ & 106 & $1.25 \pm 0.15$ \\
$\mathrm{CR} 2 \mathrm{cY}$ & $7.5 \pm 0.8$ & $12.8 \pm 0.8$ & $0.6 \pm 0.6$ & $6.6 \pm 0.7$ & $27.5 \pm 1.5$ & 29 & $1.07 \pm 0.28$ \\
\hline
\end{tabular}

Table 2. The CC search: observed yields and simulated background contributions to CRs normalized to an integrated luminosity of $35.9 \mathrm{fb}^{-1}$. The nonprompt contributions are estimated from data. The last column shows the scale factors used for the normalization of the $\mathrm{W}+$ jets and $t \overline{\mathrm{t}}$ samples. Only statistical uncertainties are reported.

samples are subject to experimental uncertainties on the jet energy scale (JES) and jet energy resolution (JER). The uncertainties due to miscalibration of the JES are estimated by varying the jet energy corrections up and down by one standard deviation and propagating the effect to the calculation of $p_{\mathrm{T}}^{\text {miss }}$. Moreover, differences of the JER between data and simulation are accounted for by smearing the momenta of jets in simulation. The uncertainties corresponding to b-tagging efficiencies and misidentification rates for tagging light-flavoured or gluon jets as b jets have been evaluated for all simulated samples. The uncertainties corresponding to the correction of simulated samples for trigger and lepton efficiencies are taken as systematic uncertainties. The uncertainty due to the simulation of pileup for simulated background processes is taken into account by varying the expected cross section of inelastic collisions by 5\% [49]. An uncertainty of $50 \%$ is assigned to the cross sections of all nonleading backgrounds. An overview of all systematic uncertainties related to the background prediction is presented in table 3 .

The nonprompt background estimation method of this search, as described in the previous section, depends on the tight-to-loose fraction $\epsilon_{\mathrm{TL}}$ which is sensitive to the flavour content of jets. The systematic uncertainty due to possible differences in the flavour content of jets between the measurement and application regions is assessed by varying the btagging requirements of the measurement region. The resulting uncertainty ranges from 20 to $50 \%$ from low to high lepton $p_{\mathrm{T}}$, respectively. The consistency of the method is tested by applying the same procedure to simulated data. To account for any residual deviation found in the test, an additional uncertainty of 20 to $200 \%$ is assigned in some regions, with the highest uncertainties applying to regions that are dominated by prompt background. 
The prompt background prediction procedure of this search, as described in the previous section, relies on the simulation of $\mathrm{W}+$ jets and $t \overline{\mathrm{t}}$ production and is sensitive to theoretical uncertainties on ISR. The modelling of ISR for these processes is checked in control samples in data that are highly enriched in t $\overline{\mathrm{t}}$ or $\mathrm{W}+$ jets events. The simulation of $t \bar{t}$ events is tested by comparing the jet multiplicity observed in a control sample with the simulation. Simulated $t \bar{t}$ events are reweighted based on this comparison, and half of the correction is assigned as the systematic uncertainty [50]. This systematic uncertainty affecting $t \bar{t}$ also affects the signal samples. Similarly, the simulation of $\mathrm{W}+$ jets events is corrected based on the distribution of $p_{\mathrm{T}}(\mathrm{W})$ in a control sample, and the difference between the uncorrected and the corrected simulation is assigned as a systematic uncertainty [51]. These two sources of uncertainties lead to relative changes of the total background estimation in the SRs that range from 2 to $10 \%$ for the $\mathrm{W}+$ jets process, and are less than $1 \%$ for the $t \bar{t}$ process. The estimate of the prompt background depends only weakly on the background composition, since the distributions of $p_{\mathrm{T}}(\ell)$ in $\mathrm{W}+$ jets and $\mathrm{t} \overline{\mathrm{t}}$ processes are similar. The corresponding systematic uncertainty is derived from a $20 \%$ variation in the relative yields of $\mathrm{W}+$ jets and $\mathrm{t} \overline{\mathrm{t}}$ backgrounds.

The dominant source of systematic uncertainty for the signal is caused by the modelling of ISR. It is minimized by reweighting the jet multiplicity in the signal sample according to the corrections obtained in the $t \bar{t}$ sample. Uncertainties due to unknown higher-order effects are estimated by variations of the renormalization and factorization scales by factors of 0.5 and 2 [52]. Moreover, possible differences between the fast and the full GEant4based modellings of $p_{\mathrm{T}}^{\text {miss }}$ are taken into account and the corresponding uncertainties are assigned to the signal yields as shown in table 3. The statistical uncertainty of the signal simulation ranges from 8 to $15 \%$.

\section{The MVA approach}

\subsection{Signal selection}

For the selection of the signal events corresponding to four-body decays of the $\widetilde{t}_{1}$, we use a boosted decision tree (BDT) $[53,54]$ to take advantage of the correlations among variables that discriminate between signal and background.

Compared to the approach of ref. [15], we use new variables and search for the most reduced set of best-performing variables to be used as input to the BDT. To find the most discriminating variables we test different sets maximizing the figure of merit (FOM) [55]:

$$
\mathrm{FOM}=\sqrt{2\left((S+B) \ln \left[\frac{(S+B)\left(B+\sigma_{B}^{2}\right)}{B^{2}+(S+B) \sigma_{B}^{2}}\right]-\left(\frac{B^{2}}{\sigma_{B}^{2}}\right) \ln \left[1+\frac{\sigma_{B}^{2} S}{B\left(B+\sigma_{B}^{2}\right)}\right]\right)},
$$

where $\mathrm{S}$ and $\mathrm{B}$ stand for the expected signal and background yields. The term $\sigma_{B}=(f B)$ represents the expected systematic uncertainty on the background with $f$ being an estimate of the relative uncertainty of the background yield, taken to be $f=20 \%$ (see section 5.3). A new variable is incorporated into the set of input variables only if it significantly increases the FOM. The full list of the final input variables is: 


\begin{tabular}{|c|c|c|c|}
\hline \multirow{2}{*}{$\begin{array}{l}\text { Systematic } \\
\text { uncertainty }\end{array}$} & \multicolumn{2}{|c|}{ Background } & \multirow[t]{2}{*}{ Signal } \\
\hline & SR1 & $\mathrm{SR} 2$ & \\
\hline \multicolumn{4}{|l|}{ Renormalization \& } \\
\hline factorization scales & - & - & $2-3$ \\
\hline Pileup & $0.1-1.8$ & $0.1-2.0$ & 1 \\
\hline JES & $1.2-2.1$ & $0.1-1.4$ & $3-4$ \\
\hline JER & $0.1-0.5$ & $0.1-1.1$ & $0-1$ \\
\hline b-tagging & 0.1 & $0.1-1.0$ & $1-3$ \\
\hline Trigger & $0.0-0.1$ & $0.0-0.1$ & 1 \\
\hline Lepton efficiency & $1.0-1.8$ & $1.0-1.5$ & 3 \\
\hline 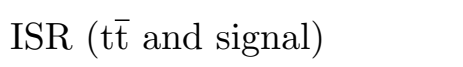 & $0.1-0.5$ & $0.1-0.8$ & $5-7$ \\
\hline ISR (W+jets) & $4.5-10.2$ & $1.9-4.4$ & - \\
\hline$p_{\mathrm{T}}^{\mathrm{miss}}$ modelling (FASTSIM) & - & - & $2-3$ \\
\hline Relative yields $\mathrm{W}+$ jets $/ \mathrm{t} \overline{\mathrm{t}}$ & $0.1-1.6$ & $0.1-2.2$ & - \\
\hline Nonprompt & $1.0-4.6$ & $1.0-9.5$ & - \\
\hline
\end{tabular}

Table 3. The CC search: typical ranges for relative systematic uncertainties (in \%) on the total background prediction and signal prediction in the main SRs. The "- " means that a certain source of uncertainty is not applicable.

- $p_{\mathrm{T}}^{\text {miss }}, p_{\mathrm{T}}(\ell)$, and $M_{\mathrm{T}}$ : the correlation between $p_{\mathrm{T}}^{\text {miss }}$ and $p_{\mathrm{T}}(\ell)$ differs between signal, where the $p_{\mathrm{T}}^{\text {miss }}$ is due to three missing objects (two $\widetilde{\chi}_{1}^{0}$ and a $\nu$ ), and $\mathrm{t} \overline{\mathrm{t}}$ and $\mathrm{W}+$ jets backgrounds where the $p_{\mathrm{T}}^{\text {miss }}$ is due to a single missing object $(\nu)$. The $M_{\mathrm{T}}$ distribution peaks at $\approx 80 \mathrm{GeV}$ for $\mathrm{SM}$ processes where a $\mathrm{W}$ boson is produced, while being a rather broad distribution for signal.

- $\eta(\ell)$ and $Q(\ell)$ : the pseudorapidity of the lepton $\eta(\ell)$ is considered because the decay products of the signal are more centrally produced than those of the $\mathrm{W}+$ jets background. The charge of the lepton $Q(\ell)$ is also considered, as $\mathrm{W}^{+}$and $\mathrm{W}^{-}$are produced unequally at the LHC, while the signal events contain equal numbers of positive and negative leptons.

- $p_{\mathrm{T}}(\mathrm{ISR}), p_{\mathrm{T}}(\mathrm{b}), N_{\text {jets }}$, and $H_{\mathrm{T}}$ : the $p_{\mathrm{T}}$ of the leading jet, $p_{\mathrm{T}}(\mathrm{ISR})$, captures the hard ISR jet in signal events, and $p_{\mathrm{T}}(\mathrm{b})$ is the $p_{\mathrm{T}}$ of the $\mathrm{b}$ jet with the highest $\mathrm{b}$ tagging discriminant value. Both are sensitive to the different phase space available for signal and background events: $m(\mathrm{t})-m(\mathrm{~W})$ for $\mathrm{t} \overline{\mathrm{t}}$, and $m\left(\widetilde{\mathrm{t}}_{1}\right)-m\left(\widetilde{\chi}_{1}^{0}\right)$ for signal. The multiplicity of selected jets $N_{\text {jets }}$ is included, reflecting the mass of the mother particle $\widetilde{t}_{1}$.

- $N\left(\mathrm{~b}^{\text {loose }}\right), \Delta R(\ell, \mathrm{b})$, and $D(\mathrm{~b})$ : the number of loose $\mathrm{b}$ jets $N\left(\mathrm{~b}^{\text {loose }}\right)$, the distance between the lepton and the jet with the highest $\mathrm{b}$ tagging discriminant $\Delta R(\ell, \mathrm{b})$, and the highest $\mathrm{b}$ tagging discriminant per event $D(\mathrm{~b})$ are also included among the input variables. 

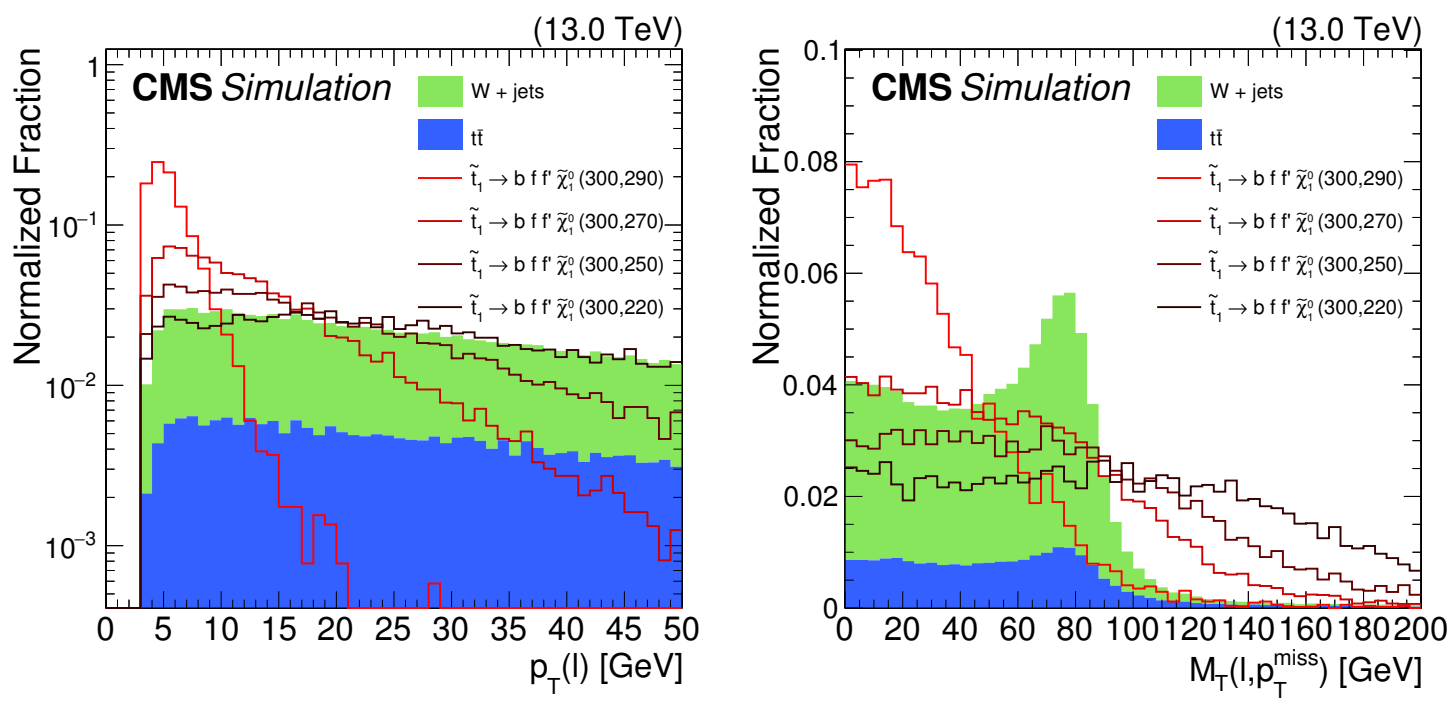

Figure 3. Simulated distributions of $p_{\mathrm{T}}(\ell)$ (left) and $M_{\mathrm{T}}$ (right) at the preselection level for signal samples with different $\Delta m$, and $\mathrm{W}+$ jets and $t \overline{\mathrm{t}}$ background events. The area of each signal distribution, and the total background contribution, are normalized to unit area.

Because the discrimination power of each input variable varies as a function of $\Delta m$, as shown in figure 3 , the $\left(m\left(\widetilde{\mathrm{t}}_{1}\right), m\left(\widetilde{\chi}_{1}^{0}\right)\right)$ plane is partitioned into eight $\Delta m$ regions (from 10 to $80 \mathrm{GeV}$, in $10 \mathrm{GeV}$ steps) and a separate BDT is trained for each partition. The $\mathrm{W}+$ jets and $\mathrm{t} \overline{\mathrm{t}}$ processes, which represent a large fraction of the total background after preselection, are included in the training of the $\mathrm{BDT}$. The $\mathrm{Z}(\rightarrow \nu \nu)+$ jets process, which represents a nonnegligible fraction of the remaining total background at the final selection level, is also included. The training is done with simulated events for both signal and background processes. The background samples are normalized to their respective cross section to realistically represent the SM background in the training. We take advantage of the similar distribution of the input variables for different $\left(m\left(\widetilde{\mathrm{t}}_{1}\right), m\left(\widetilde{\chi}_{1}^{0}\right)\right)$ signal points with the same $\Delta m$, and regroup all signal points for a given $\Delta m$ together when feeding signal to the BDT training. This increases the number of signal events for each training. Due to the large variation of the spectrum of the $p_{\mathrm{T}}(\ell)$ variable across the $\left(m\left(\widetilde{\mathrm{t}}_{1}\right), m\left(\widetilde{\chi}_{1}^{0}\right)\right)$ plane, we require $p_{\mathrm{T}}(\ell)<30 \mathrm{GeV}$ for signal points with $\Delta m \leq 60 \mathrm{GeV}$ before training different BDTs, while there is no restriction on $p_{T}(\ell)$ for signal points with higher $\Delta m$.

Figures 4 and 5 show the output distribution of the BDT in data and for the total SM background as taken from simulation. In each case a representative $\left(m\left(\widetilde{\mathrm{t}}_{1}\right), m\left(\widetilde{\chi}_{1}^{0}\right)\right)$ signal point is also shown, chosen at the limit of the expected sensitivity of the CC search (see section 6) and belonging to the $\Delta m$ for which the training has been done. We observe that the responses of the BDT, henceforth called BDT outputs, are not the same. This is due to the changing mix between signal and background as well as varying differences of correlations across the $\left.\left(m \widetilde{\mathrm{t}}_{1}\right), m\left(\widetilde{\chi}_{1}^{0}\right)\right)$ plane, resulting in different BDT outputs for different $\Delta m$ values. We observe good agreement between data and simulation over the entire range dominated by the background (e.g. BDT output smaller than 0.3) for the eight different 
trainings. The BDT output is also checked in data to be well reproduced by the simulation in two validation regions, across the entire range of the BDT output. These regions are kinematically orthogonal to the preselection while using the same online selection, and are defined as follows:

- Preselection with $200<p_{\mathrm{T}}^{\text {miss }}<280 \mathrm{GeV}$,

- Preselection with $p_{\mathrm{T}}(\ell)>30 \mathrm{GeV}$.

They are also used to evaluate the precision of the method for predicting background, as described in section 5.2. A SR is defined by applying a threshold to each BDT output. The thresholds on the BDT output are reported in table 5 . On average the BDT selection suppresses the SM background by a factor $\approx 3 \times 10^{3}$ while reducing the signal by a factor $\approx 25$. The total efficiency for signal points at the limit of the sensitivity of the CC search, and across all selections, is of the order of $1.3 \times 10^{-4}$.

\subsection{Background predictions}

The predicted numbers of $\mathrm{W}+$ jets and $\mathrm{t} \overline{\mathrm{t}}$ events are obtained from data control regions (CRs) based on the BDT output. The number of estimated prompt background events in the SR, $Y_{\text {prompt }}^{\mathrm{SR}}$, is derived as follows:

$$
Y_{\text {prompt }}^{\mathrm{SR}}(X)=N_{\text {prompt }}^{\mathrm{SR}}(X)\left[\frac{N^{\mathrm{CR}}(\text { Data })-N_{\text {prompt }}^{\mathrm{CR}}(\text { Rare })-N_{\text {nonprompt }}^{\mathrm{CR}}}{N_{\text {prompt }}^{\mathrm{CR}}(X)}\right] .
$$

Here, $X$ refers to background processes to be estimated, $\mathrm{W}+$ jets or $t \overline{\mathrm{t}}$. The superscripts $\mathrm{SR}$ and CR, respectively, refer to the signal and control regions. The term "prompt" refers to processes where a prompt lepton is produced. The term "nonprompt" refers to processes where there is a jet misreconstructed as a lepton. The numbers $N_{\text {prompt }}^{\mathrm{SR}, \mathrm{CR}}(X)$ are predicted from simulated background. The number $N_{\text {prompt }}^{\mathrm{CR}}$ (Rare) refers to simulated background processes other than those being estimated, and includes single top, DY, and diboson production. The number $N_{\text {nonprompt }}^{\mathrm{CR}}$ refers to the estimate of the backgrounds with a nonprompt lepton from data (as explained in section 4.2). Within the region defined by the preselection, the $\mathrm{CRs}$ are obtained by requiring $\mathrm{BDT}<0.2$ to get a data sample enriched in background. They are further enriched in $t \bar{t}$ events by requiring them to have at least one tight $\mathrm{b}$ jet, and in $\mathrm{W}+$ jets events by requiring the number of loose $\mathrm{b}$ jets to be zero. The level of potential signal contamination in the CRs is well below $5 \%$ and is not expected to impact the final result.

The systematic uncertainties associated with these predictions are based on differences between the predicted number of events (obtained from eq. (5.2)) and the observed number of data events, both in validation regions as defined in the previous section.

The number $Y_{\text {nonprompt }}^{\text {SR }}$ of background events with nonprompt leptons is estimated from data in all signal regions with the method described in section 4.2. The yield of other SM processes such as diboson, single top, and DY production are estimated from simulation. 

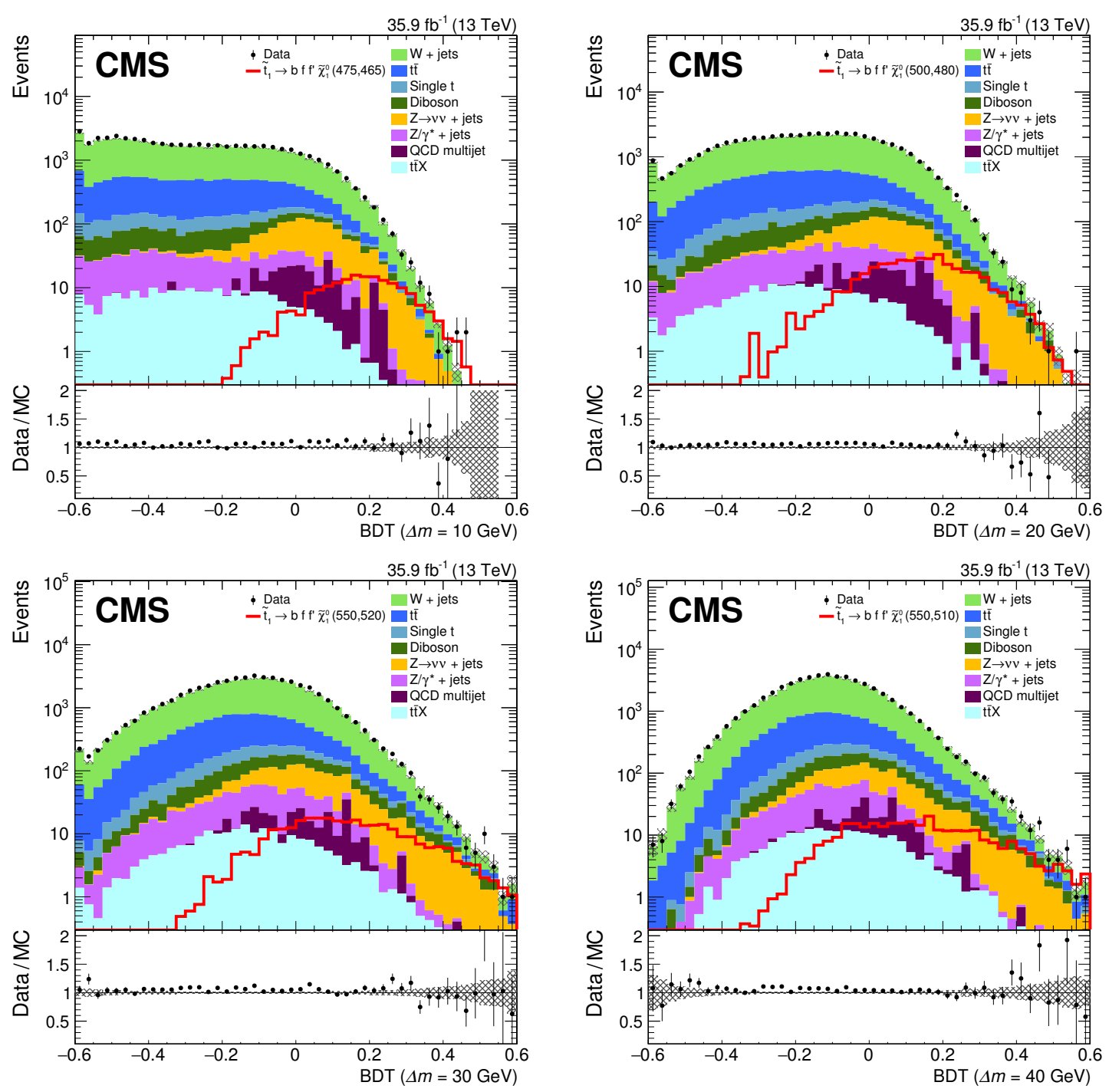

Figure 4. Distributions of the BDT output at the preselection level in data and simulation in $10 \mathrm{GeV}$ steps of $\Delta m$ from 10 (top-left) to $40 \mathrm{GeV}$ (bottom-right). For each case, a representative $\left(m\left(\widetilde{\mathrm{t}}_{1}\right), m\left(\widetilde{\chi}_{1}^{0}\right)\right)$ signal point is also shown, but is not added to the SM background. The shaded area on the Data/MC ratio represents the statistical uncertainty of the simulated background.

\subsection{Systematic uncertainties}

All processes that are modelled by simulation are subject to the same systematic uncertainties as described in section 4.3. The statistical uncertainty of the signal simulation ranges between 3 and $11 \%$. The systematic uncertainty affecting the prediction of the $\mathrm{W}+$ jets and $\mathrm{t} \overline{\mathrm{t}}$ backgrounds has been described in section 5.2, where the statistical uncertainty from the number of events in CRs is included. The uncertainties are evaluated from both validation regions, and the larger value is conservatively chosen. Furthermore, uncertainties on the shape of the BDT output, which can affect the background prediction, have been assessed. They are smaller than the aforementioned systematic uncertainties. The systematic un- 

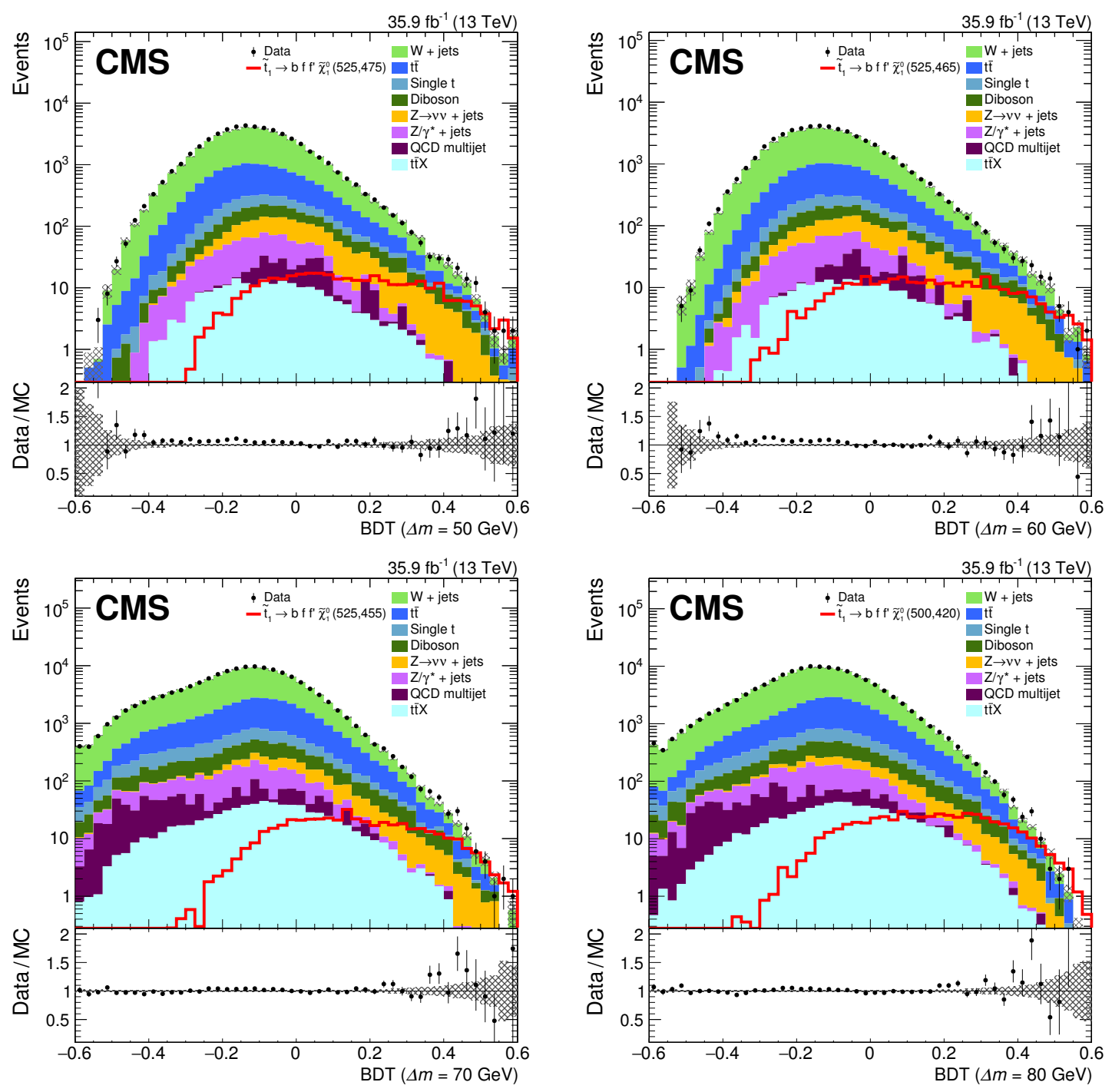

Figure 5. Distributions of the BDT output at the preselection level in data and simulation in $10 \mathrm{GeV}$ steps of $\Delta m$ from 50 (top-left) to $80 \mathrm{GeV}$ (bottom-right). For each case, a representative $\left(m\left(\widetilde{\mathrm{t}}_{1}\right), m\left(\widetilde{\chi}_{1}^{0}\right)\right)$ signal point is also shown, but is not added to the SM background. The shaded area on the Data/MC ratio represents the statistical uncertainty of the simulated background.

certainties affecting the prediction of the nonprompt lepton background are the same as in section 4.2. As we perform a separate analysis for each $\Delta m$ region, the uncertainties are evaluated separately and can therefore vary across different values of $\Delta m$. The relative systematic uncertainties on the predictions of the $\mathrm{W}+$ jets, $t \overline{\mathrm{t}}$, and nonprompt lepton on the total background are provided in table 4 .

\section{Results}

After performing the two searches, we find no evidence for direct top squark production, as can be seen in table 5 and in figure 6 for the MVA and CC searches, respectively. Both 


\begin{tabular}{|c|c|c|}
\hline $\begin{array}{l}\text { Systematic } \\
\text { uncertainty }\end{array}$ & Background & Signal \\
\hline \multicolumn{3}{|l|}{ Renormalization \& } \\
\hline factorization scales & $0-1$ & $1.5-3.0$ \\
\hline Pileup & 1 & 1 \\
\hline JES & $0-2$ & $6-13$ \\
\hline JER & $0-1$ & $1-6$ \\
\hline b-tagging & $0-1$ & $0-7$ \\
\hline Trigger & 1 & 1 \\
\hline Lepton efficiency & $0-1$ & 4 \\
\hline 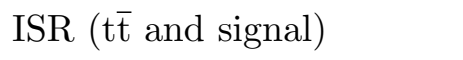 & $0-1$ & $0-6$ \\
\hline ISR (W+jets) & $0-10$ & - \\
\hline$p_{\mathrm{T}}^{\mathrm{miss}}$ modelling (FASTSIM) & - & 5 \\
\hline Prediction of $\mathrm{W}+$ jets & $7.1-32.0$ & - \\
\hline Prediction of $t \bar{t}$ & $4.1-16.0$ & - \\
\hline Prediction of nonprompt & $6.7-15.6$ & - \\
\hline
\end{tabular}

Table 4. The MVA search: relative systematic uncertainties (in \%) on the total background and signal prediction. The "- means that a certain source of uncertainty is not applicable. In the case of the background, the uncertainties are on the total background. Systematic uncertainties on the data-driven prediction of the $\mathrm{W}+\mathrm{jets}, \mathrm{t} \overline{\mathrm{t}}$, and nonprompt lepton backgrounds are reported.

sets of results include the prediction of the $\mathrm{W}+\mathrm{jets}$ and $t \overline{\mathrm{t}}$ processes, the prediction of the background with a nonprompt lepton, the prediction of other background processes from simulation, the total expected background, and the observed number of data events. Systematic uncertainties are included in the predictions. For the MVA search (table 5), the overlap between the SRs defined for different $\Delta m$ is generally below $50 \%$ for adjacent regions, and ranges from 0 to $30 \%$ for nonadjacent regions. Taking into account these results, the expected signal yield for each $\left(m\left(\widetilde{\mathrm{t}}_{1}\right), m\left(\widetilde{\chi}_{1}^{0}\right)\right)$ mass point, and the corresponding systematic uncertainties, we interpret the absence of a clear excess in terms of a $95 \%$ confidence level (CL) exclusion of top squark pair production in the $\left(m\left(\widetilde{\mathrm{t}}_{1}\right), m\left(\widetilde{\chi}_{1}^{0}\right)\right)$ plane. The limits are calculated according to the modified frequentist $\mathrm{CL}_{\mathrm{s}}$ criterion [56-58]. A test statistic, defined to be the likelihood ratio between the background-only and signalplus-background hypotheses, is used to set exclusion limits on top squark pair production. For the CC search, which features a larger number of signal regions, an asymptotic approximation [55] is used, while in the MVA search the distributions of these test statistics are constructed using simulated experiments. Statistical uncertainties are modelled as Poisson distributions. All systematic uncertainties are modelled with a log-normal distribution. In the CC search, the effect of signal contamination in the CRs is taken into account by including the control regions, with the estimate of corresponding signal yields, in the like- 
lihood fit. When interpreting the results, we assume branching fractions of $100 \%$ for the two considered decay scenarios.

Figure 7 represents the exclusion contour as a function of $m\left(\widetilde{\mathrm{t}}_{1}\right)$ and $\Delta m$ for both searches in the case of the four-body decay scenario. For this decay mode, the CC search reaches its highest mass exclusion of $500 \mathrm{GeV}$ for $\Delta m \approx 30 \mathrm{GeV}$. At $\Delta m=80 \mathrm{GeV}$, the analysis probes masses up to $390 \mathrm{GeV}$. For the MVA search, the maximum sensitivity is reached for the highest $\Delta m$ of $80 \mathrm{GeV}$, where top squark masses up to $560 \mathrm{GeV}$ are excluded. At $\Delta m=10 \mathrm{GeV}$, the corresponding value is $420 \mathrm{GeV}$. For both analyses, the reduced sensitivity at the lowest mass differences is due to the decrease in the transverse momenta of the visible decay products, as shown in figure 3 , and the corresponding loss in acceptance. For intermediate values of $\Delta m$, the two approaches obtain similar limits. At the highest mass differences, the MVA selection has higher acceptance than the CC approach as it also includes events with lepton $p_{\mathrm{T}}>30 \mathrm{GeV}$ while keeping the level of background under control.

Figure 8 represents the interpretation of the CC search for the chargino-mediated scenario. For this model, with a chargino mass equal to the average of the top squark and neutralino masses, the sensitivity is very similar to the case of four-body decays, with the maximum exclusion being reached at slightly higher values of $\Delta m$. Top squark masses of up to $540 \mathrm{GeV}$ are excluded for $\Delta m \approx 40 \mathrm{GeV}$.

In order to constrain top squark pair production in both decay modes using the information from several final states, a statistical combination of the CC search with the all-hadronic search [19] for both decay scenarios of the top squark is performed. The common systematic uncertainties of the two searches are treated as fully correlated, and the possible correlations arising from events passing the selection criteria of both searches are found to have negligible impact on the final results. The combined limits, shown in figure 9, include all SRs and CRs of the all-hadronic and the single-lepton CC searches. The combination of the two searches extends the exclusion limits on the top squark mass up to 590 and $670 \mathrm{GeV}$ for the four-body and chargino-mediated scenarios, respectively.

\section{Summary}

A search for direct top squark pair production is performed in a compressed scenario where the mass difference $\Delta m$ between the lightest top squark and the lightest supersymmetric particle (LSP), taken to be the lightest neutralino $\widetilde{\chi}_{1}^{0}$, does not exceed the $\mathrm{W}$ boson mass. Two decay modes of the top squark are targeted: the four-body prompt decay to $\mathrm{bf}^{\prime} \widetilde{\chi}_{1}^{0}$, and the chargino-mediated decay to $\mathrm{b} \widetilde{\chi}_{1}^{+}$with a subsequent decay $\widetilde{\chi}_{1}^{+} \rightarrow \mathrm{f} \overline{\mathrm{f}}^{\prime} \widetilde{\chi}_{1}^{0}$. Results are based on proton-proton collision data at $\sqrt{s}=13 \mathrm{TeV}$, recorded with the CMS detector in 2016 and corresponding to an integrated luminosity of $35.9 \mathrm{fb}^{-1}$. Selected events are required to have a single lepton (electron or muon), and significant missing transverse momentum $\left(p_{\mathrm{T}}^{\text {miss }}\right)$. Because of the small mass difference between the top squark and the LSP, the decay products of the top squark are expected to have low $p_{\mathrm{T}}$. Events where the presence of a jet from initial-state radiation leads to a boost of the top squark pair and sizeable $p_{\mathrm{T}}^{\text {miss }}$ are selected. 


\begin{tabular}{|c|c|c|c|c|c|c|c|}
\hline & BDT> & $\begin{array}{c}Y_{\text {prompt }}^{\mathrm{SR}} \\
(\mathrm{W}+\text { jets })\end{array}$ & $\begin{array}{c}Y_{\text {prompt }}^{\mathrm{SR}} \\
(\mathrm{t} \overline{\mathrm{t}})\end{array}$ & $Y_{\text {nonprompt }}^{\mathrm{SR}}$ & $\begin{array}{c}N^{\mathrm{SR}} \\
\text { (Rare) }\end{array}$ & $N^{\mathrm{SR}}(\mathrm{B})$ & $N^{\mathrm{SR}}(\mathrm{D})$ \\
\hline$\Delta m=10 \mathrm{GeV}$ & 0.31 & $18.4 \pm 3.6$ & $1.8 \pm 4.8$ & $8.0 \pm 2.9$ & $2.3 \pm 1.4$ & $30.3 \pm 6.7$ & 39 \\
\hline$\Delta m=20 \mathrm{GeV}$ & 0.39 & $9.0 \pm 2.0$ & $1.3 \pm 1.7$ & $11.2 \pm 3.2$ & $3.1 \pm 1.9$ & $24.7 \pm 4.5$ & 20 \\
\hline$\Delta m=30 \mathrm{GeV}$ & 0.47 & $4.0 \pm 2.5$ & $1.2 \pm 0.6$ & $8.8 \pm 2.5$ & $1.7 \pm 1.2$ & $15.7 \pm 3.7$ & 22 \\
\hline$\Delta m=40 \mathrm{GeV}$ & 0.48 & $4.1 \pm 1.3$ & $1.8 \pm 0.7$ & $7.6 \pm 2.3$ & $1.2 \pm 0.9$ & $14.8 \pm 2.8$ & 16 \\
\hline$\Delta m=50 \mathrm{GeV}$ & 0.45 & $7.3 \pm 2.1$ & $4.7 \pm 2.8$ & $7.1 \pm 2.0$ & $5.5 \pm 3.1$ & $24.5 \pm 4.8$ & 36 \\
\hline$\Delta m=60 \mathrm{GeV}$ & 0.50 & $2.0 \pm 0.6$ & $2.4 \pm 1.2$ & $3.1 \pm 1.1$ & $1.1 \pm 0.9$ & $8.7 \pm 1.8$ & 12 \\
\hline$\Delta m=70 \mathrm{GeV}$ & 0.46 & $4.9 \pm 1.6$ & $3.4 \pm 1.1$ & $5.4 \pm 1.6$ & $3.2 \pm 1.9$ & $16.8 \pm 2.9$ & 20 \\
\hline$\Delta m=80 \mathrm{GeV}$ & 0.44 & $7.1 \pm 1.6$ & $5.1 \pm 0.9$ & $5.3 \pm 1.6$ & $5.2 \pm 3.0$ & $22.8 \pm 3.3$ & 26 \\
\hline
\end{tabular}

Table 5. The MVA search: prediction of the $\mathrm{W}+$ jets, $\mathrm{t} \overline{\mathrm{t}}$, nonprompt lepton, and other backgrounds in the eight SRs defined by the threshold on the BDT output reported in the second column. The prediction of the first three processes is based on data, while that of $N^{S R}$ (Rare), i.e. rare backgrounds, is based on simulation. The uncertainties are the quadrature sum of the statistical uncertainties, the systematic uncertainties of table 4, and for the backgrounds predicted from simulation, the cross section uncertainties. The number of total expected background $\left(N^{S R}(\mathrm{~B})\right)$ and observed data $\left(N^{S R}(\mathrm{D})\right)$ events in each $\mathrm{SR}$ are also reported.

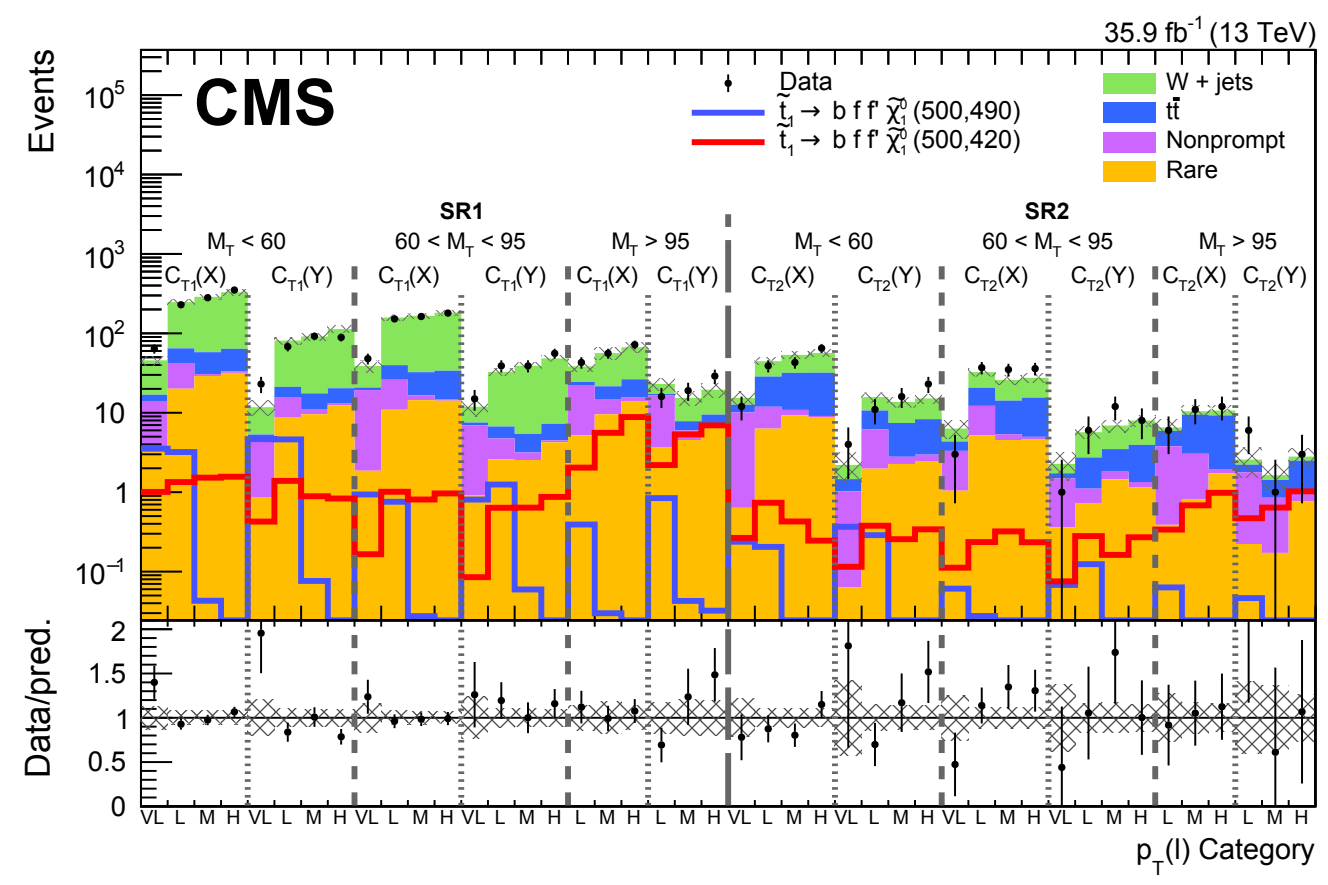

Figure 6. The CC approach: summary of observed and expected background yields in all SRs as defined in table 1. The vertical bars and the shaded areas represent the statistical uncertainty of the data and the total uncertainty in the prediction, respectively. The lower panel shows the ratio of data to prediction. 

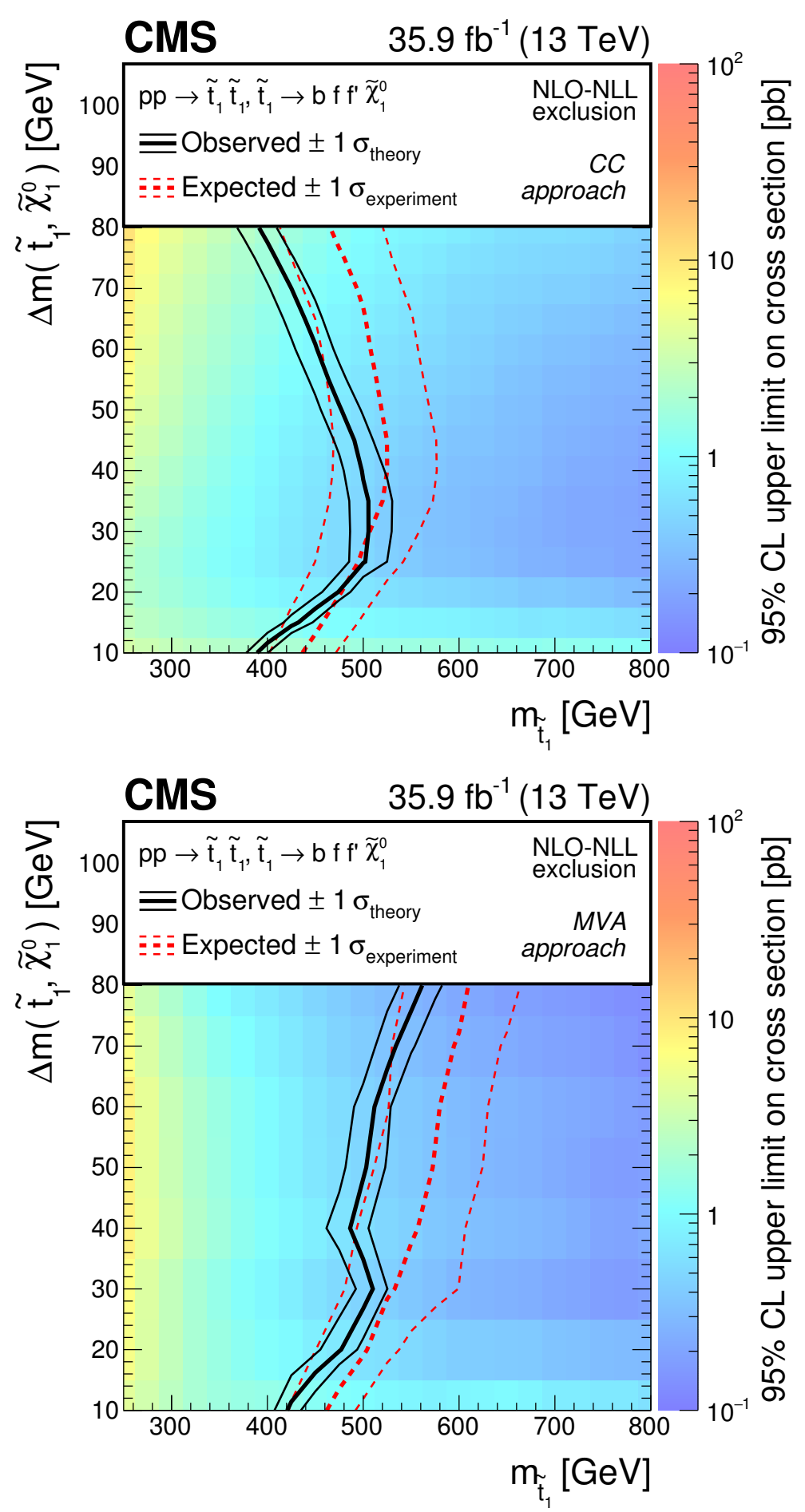

Figure 7. Exclusion limit at 95\% CL for the four-body decay of the top squark as a function of $m\left(\widetilde{\mathrm{t}}_{1}\right)$ and $\Delta m$ for the CC (upper) and MVA (lower) approaches. The colour shading corresponds to the observed limit on the cross section. The solid black (dashed red) lines represent the observed (expected) limits, derived using the expected top squark pair production cross section. The thick lines represent the central values and the thin lines the variations due to the theoretical or experimental uncertainties. 


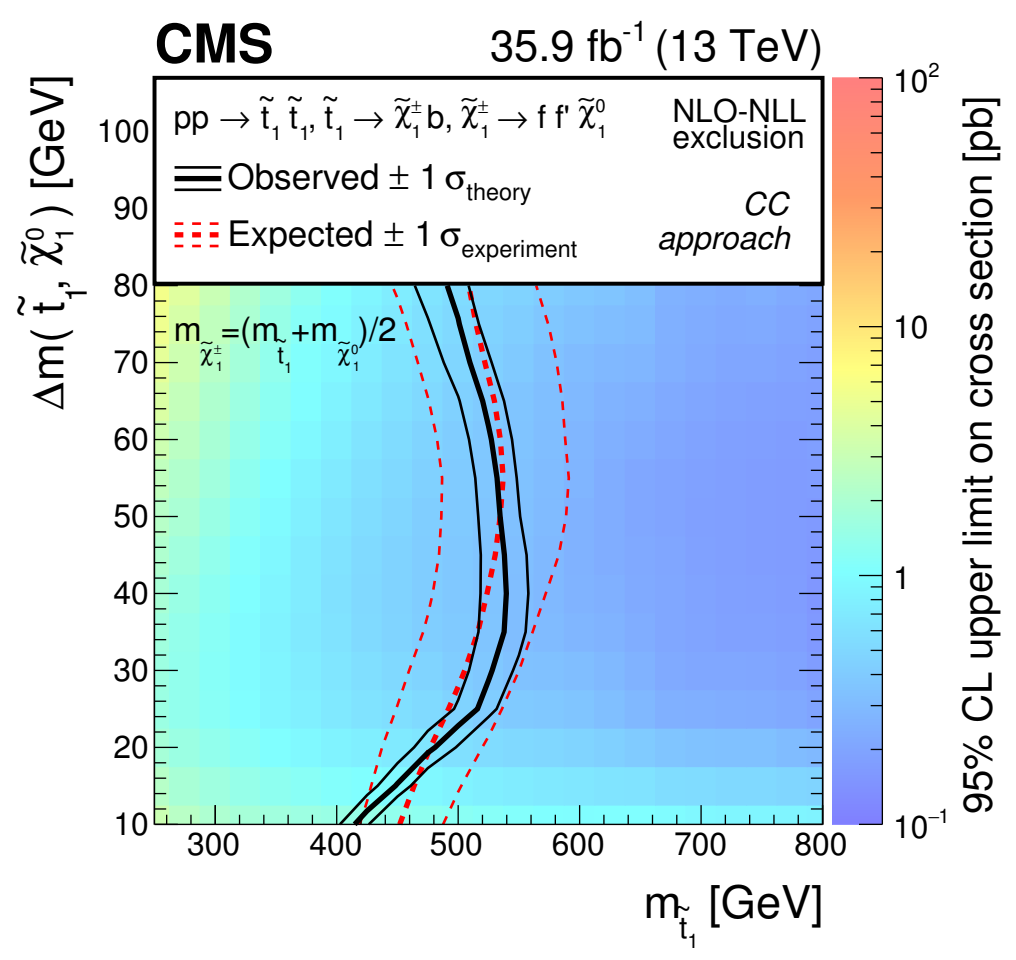

Figure 8. Exclusion limit at 95\% CL for the chargino-mediated decay of the top squark as a function of $m\left(\widetilde{\mathrm{t}}_{1}\right)$ and $\Delta m$ for the CC search. The colour shading corresponds to the observed limit on the cross section. The solid black (dashed red) lines represent the observed (expected) limits, derived using the expected top squark pair production cross section. The thick lines represent the central values and the thin lines the variations due to the theoretical (experimental) uncertainties.

Two search strategies are pursued. In the sequential selection approach (CC), signal regions are defined based on discriminating variables, particularly the transverse mass of the lepton- $p_{\mathrm{T}}^{\text {miss }}$ system and the lepton momentum. In another approach, a multivariate analysis (MVA) is employed that uses both kinematic and topological variables and is specifically trained for different $\Delta m$ regions of the four-body decay mode. In both approaches, the dominant contributions to the signal regions from standard model processes $(\mathrm{W}+\mathrm{jets}, \mathrm{t} \overline{\mathrm{t}}$, and events with misidentified leptons) are estimated from control regions in data.

Data are found to be compatible with the predicted standard model backgrounds. The results are used to set limits at $95 \%$ confidence level on the production cross section as a function of the $\widetilde{t}_{1}$ and $\widetilde{\chi}_{1}^{0}$ masses, within the context of simplified models. Assuming $100 \%$ branching fraction in the decay channel under consideration and the top squark pair production cross section computed at NLO+NLL precision [40-46], these limits are converted into mass limits.

Both search strategies are applied to the four-body decay mode. For this decay mode, the MVA search excludes top squark masses up to 420 and $560 \mathrm{GeV}$ at $\Delta m=10$ and $80 \mathrm{GeV}$, respectively. The $\mathrm{CC}$ approach covers the chargino-mediated decays, where the chargino mass is taken as the average of the top squark and the neutralino masses, probing $\widetilde{t}_{1}$ masses 

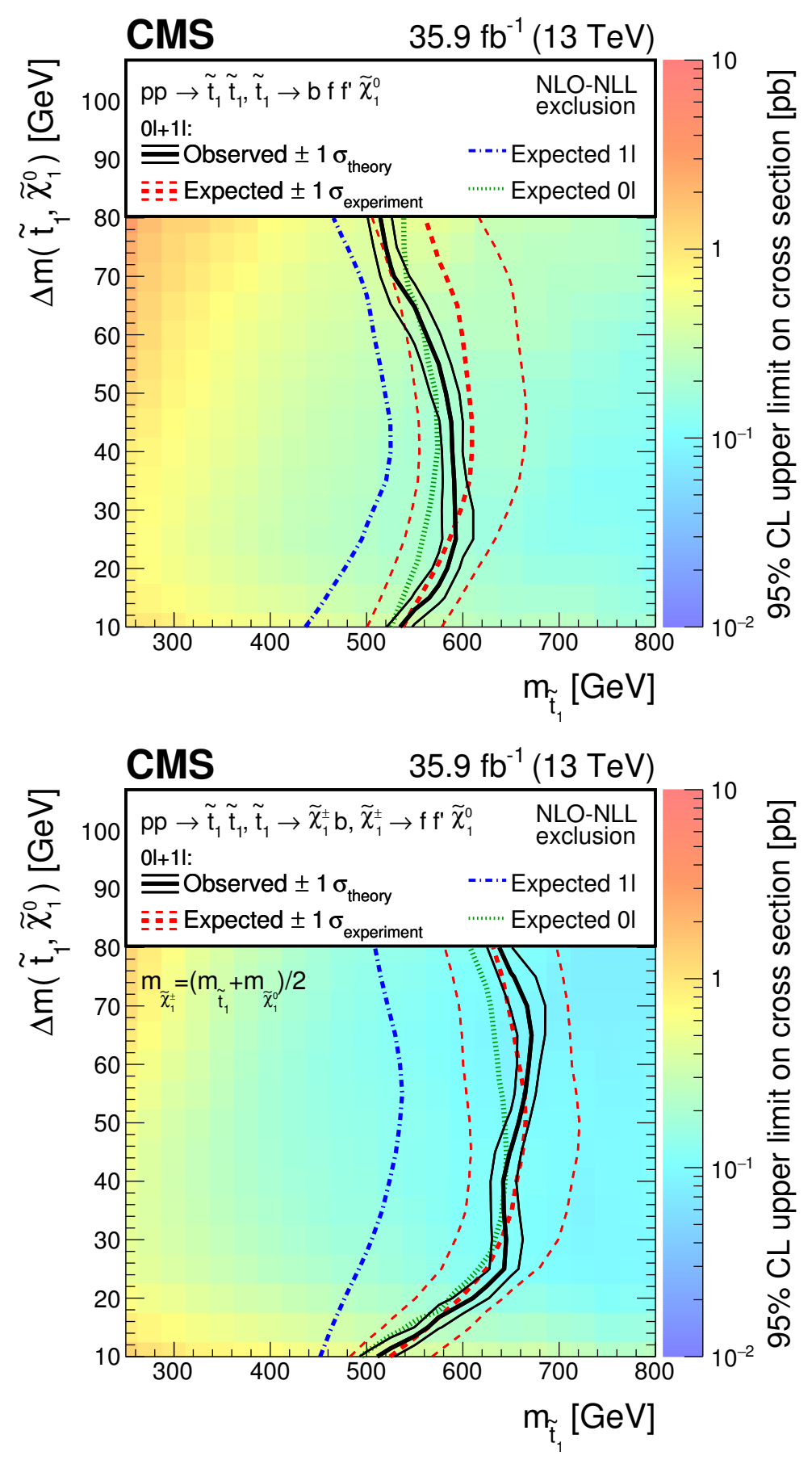

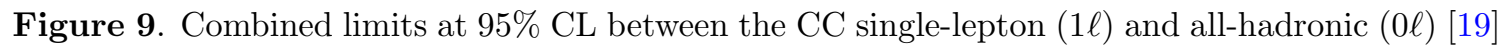
searches for the four-body decay (upper) and the chargino-mediated decay (lower) of the top squark in the $m\left(\widetilde{\mathrm{t}}_{1}\right)-\Delta m\left(\widetilde{\mathrm{t}}_{1}, \widetilde{\chi}_{1}^{0}\right)$ plane. The correlations between the two searches have been taken into account. The colour shading corresponds to the observed limit on the cross section. The solid black (dashed red) lines show the observed (expected) mass limits, derived using the expected top squark pair production cross section. The thick lines represent the central values and the thin lines the variations due to the theoretical (experimental) uncertainties. The dot-dashed blue and dotted green lines show the individual expected mass limits for the $1 \ell$ and $0 \ell$ searches, respectively. 
up to $540 \mathrm{GeV}$ for $\Delta m \approx 40 \mathrm{GeV}$. The results of the $\mathrm{CC}$ search have been combined with a search for top squark pair production in the fully hadronic channel [19]. The combined mass limits reach up to 590 and $670 \mathrm{GeV}$ for four-body and chargino-mediated decays, respectively. The reach of the $\Delta m$ dependent MVA search in the four-body decay mode is noteworthy, as the exclusion limit goes beyond that of the combined result at high $\Delta m$.

The results summarized in this paper represent the most stringent limits to date on the top squark pair production cross section for mass differences between the top squark and the lightest neutralino below the $\mathrm{W}$ boson mass, and for decays proceeding through the four-body or the chargino-mediated modes.

\section{Acknowledgments}

We congratulate our colleagues in the CERN accelerator departments for the excellent performance of the LHC and thank the technical and administrative staffs at CERN and at other CMS institutes for their contributions to the success of the CMS effort. In addition, we gratefully acknowledge the computing centres and personnel of the Worldwide LHC Computing Grid for delivering so effectively the computing infrastructure essential to our analyses. Finally, we acknowledge the enduring support for the construction and operation of the LHC and the CMS detector provided by the following funding agencies: BMWFW and FWF (Austria); FNRS and FWO (Belgium); CNPq, CAPES, FAPERJ, and FAPESP (Brazil); MES (Bulgaria); CERN; CAS, MoST, and NSFC (China); COLCIENCIAS (Colombia); MSES and CSF (Croatia); RPF (Cyprus); SENESCYT (Ecuador); MoER, ERC IUT, and ERDF (Estonia); Academy of Finland, MEC, and HIP (Finland); CEA and CNRS/IN2P3 (France); BMBF, DFG, and HGF (Germany); GSRT (Greece); NKFIA (Hungary); DAE and DST (India); IPM (Iran); SFI (Ireland); INFN (Italy); MSIP and NRF (Republic of Korea); LAS (Lithuania); MOE and UM (Malaysia); BUAP, CINVESTAV, CONACYT, LNS, SEP, and UASLP-FAI (Mexico); MBIE (New Zealand); PAEC (Pakistan); MSHE and NSC (Poland); FCT (Portugal); JINR (Dubna); MON, RosAtom, RAS and RFBR (Russia); MESTD (Serbia); SEIDI, CPAN, PCTI and FEDER (Spain); Swiss Funding Agencies (Switzerland); MST (Taipei); ThEPCenter, IPST, STAR, and NSTDA (Thailand); TUBITAK and TAEK (Turkey); NASU and SFFR (Ukraine); STFC (United Kingdom); DOE and NSF (U.S.A.).

Individuals have received support from the Marie-Curie programme and the European Research Council and Horizon 2020 Grant, contract No. 675440 (European Union); the Leventis Foundation; the A. P. Sloan Foundation; the Alexander von Humboldt Foundation; the Belgian Federal Science Policy Office; the Fonds pour la Formation à la Recherche dans l'Industrie et dans l'Agriculture (FRIA-Belgium); the Agentschap voor Innovatie door Wetenschap en Technologie (IWT-Belgium); the F.R.S.-FNRS and FWO (Belgium) under the "Excellence of Science - EOS" — be.h project n. 30820817; the Ministry of Education, Youth and Sports (MEYS) of the Czech Republic; the Lendület ("Momentum") Programme and the János Bolyai Research Scholarship of the Hungarian Academy of Sciences, the New National Excellence Program ÚNKP, the NKFIA research grants 123842, 123959, 124845, 124850 and 125105 (Hungary); the Council of Science and Industrial Research, 
India; the HOMING PLUS programme of the Foundation for Polish Science, cofinanced from European Union, Regional Development Fund, the Mobility Plus programme of the Ministry of Science and Higher Education, the National Science Center (Poland), contracts Harmonia 2014/14/M/ST2/00428, Opus 2014/13/B/ST2/02543, 2014/15/B/ST2/03998, and 2015/19/B/ST2/02861, Sonata-bis 2012/07/E/ST2/01406; the National Priorities Research Program by Qatar National Research Fund; the Programa Estatal de Fomento de la Investigación Científica y Técnica de Excelencia María de Maeztu, grant MDM-2015-0509 and the Programa Severo Ochoa del Principado de Asturias; the Thalis and Aristeia programmes cofinanced by EU-ESF and the Greek NSRF; the Rachadapisek Sompot Fund for Postdoctoral Fellowship, Chulalongkorn University and the Chulalongkorn Academic into Its 2nd Century Project Advancement Project (Thailand); the Welch Foundation, contract C-1845; and the Weston Havens Foundation (U.S.A.).

Open Access. This article is distributed under the terms of the Creative Commons Attribution License (CC-BY 4.0), which permits any use, distribution and reproduction in any medium, provided the original author(s) and source are credited.

\section{References}

[1] S.P. Martin, A supersymmetry primer, Adv. Ser. Direct. High Energy Phys. 18 (1998) 1 [Adv. Ser. Direct. High Energy Phys. 21 (2010) 1] [hep-ph/9709356] [INSPIRE].

[2] J. Wess and B. Zumino, Supergauge transformations in four-dimensions, Nucl. Phys. B 70 (1974) 39 [INSPIRE].

[3] H.P. Nilles, Supersymmetry, supergravity and particle physics, Phys. Rept. 110 (1984) 1 [INSPIRE].

[4] H.E. Haber and G.L. Kane, The search for supersymmetry: probing physics beyond the Standard Model, Phys. Rept. 117 (1985) 75 [InSPIRE].

[5] R. Barbieri, S. Ferrara and C.A. Savoy, Gauge models with spontaneously broken local supersymmetry, Phys. Lett. B 119 (1982) 343 [InSPIRE].

[6] S. Dawson, E. Eichten and C. Quigg, Search for supersymmetric particles in hadron-hadron collisions, Phys. Rev. D 31 (1985) 1581 [INSPIRE].

[7] G.R. Farrar and P. Fayet, Phenomenology of the production, decay and detection of new hadronic states associated with supersymmetry, Phys. Lett. B 76 (1978) 575 [INSPIRE].

[8] E. Witten, Dynamical breaking of supersymmetry, Nucl. Phys. B 188 (1981) 513 [InSPIRE].

[9] S. Dimopoulos and H. Georgi, Softly broken supersymmetry and SU(5), Nucl. Phys. B 193 (1981) 150 [INSPIRE].

[10] ATLAS collaboration, Observation of a new particle in the search for the Standard Model Higgs boson with the ATLAS detector at the LHC, Phys. Lett. B 716 (2012) 1 [arXiv: 1207.7214] [INSPIRE].

[11] CMS collaboration, Observation of a new boson at a mass of $125 \mathrm{GeV}$ with the CMS experiment at the LHC, Phys. Lett. B 716 (2012) 30 [arXiv:1207.7235] [INSPIRE].

[12] CMS collaboration, Observation of a new boson with mass near $125 \mathrm{GeV}$ in pp collisions at $\sqrt{s}=7$ and $8 \mathrm{TeV}$, JHEP 06 (2013) 081 [arXiv:1303.4571] [INSPIRE]. 
[13] C. Balázs, M. Carena and C.E.M. Wagner, Dark matter, light stops and electroweak baryogenesis, Phys. Rev. D 70 (2004) 015007 [hep-ph/0403224] [INSPIRE].

[14] T. Cohen et al., SUSY simplified models at 14, 33 and $100 \mathrm{TeV}$ proton colliders, JHEP 04 (2014) 117 [arXiv:1311.6480] [INSPIRE].

[15] CMS collaboration, Search for supersymmetry in events with soft leptons, low jet multiplicity and missing transverse energy in proton-proton collisions at $\sqrt{s}=8 \mathrm{TeV}$, Phys. Lett. B 759 (2016) 9 [arXiv: 1512.08002] [INSPIRE].

[16] ATLAS collaboration, Search for top squark pair production in final states with one isolated lepton, jets and missing transverse momentum in $\sqrt{s}=8 \mathrm{TeV}$ pp collisions with the ATLAS detector, JHEP 11 (2014) 118 [arXiv:1407.0583] [INSPIRE].

[17] ATLAS collaboration, Search for top-squark pair production in final states with one lepton, jets and missing transverse momentum using $36 \mathrm{fb}^{-1}$ of $\sqrt{s}=13 \mathrm{TeV}$ pp collision data with the ATLAS detector, JHEP 06 (2018) 108 [arXiv:1711.11520] [INSPIRE].

[18] ATLAS collaboration, Search for dark matter and other new phenomena in events with an energetic jet and large missing transverse momentum using the ATLAS detector, JHEP 01 (2018) 126 [arXiv: 1711.03301] [INSPIRE].

[19] CMS collaboration, Search for direct production of supersymmetric partners of the top quark in the all-jets final state in proton-proton collisions at $\sqrt{s}=13 \mathrm{TeV}$, JHEP 10 (2017) 005 [arXiv: 1707.03316] [INSPIRE].

[20] ATLAS collaboration, Search for direct top squark pair production in final states with two leptons in $\sqrt{s}=13 \mathrm{TeV}$ pp collisions with the ATLAS detector, Eur. Phys. J. C 77 (2017) 898 [arXiv: 1708.03247] [INSPIRE].

[21] CMS collaboration, Search for new physics in events with two soft oppositely charged leptons and missing transverse momentum in proton-proton collisions at $\sqrt{s}=13 \mathrm{TeV}$, Phys. Lett. B 782 (2018) 440 [arXiv: 1801.01846] [INSPIRE].

[22] CMS collaboration, The CMS experiment at the CERN LHC, 2008 JINST 3 S08004 [INSPIRE].

[23] CMS collaboration, Particle-flow reconstruction and global event description with the CMS detector, 2017 JINST 12 P10003 [arXiv:1706. 04965] [INSPIRE].

[24] M. Cacciari, G.P. Salam and G. Soyez, The anti- $k_{t}$ jet clustering algorithm, JHEP 04 (2008) 063 [arXiv: 0802.1189] [INSPIRE].

[25] M. Cacciari, G.P. Salam and G. Soyez, FastJet user manual, Eur. Phys. J. C 72 (2012) 1896 [arXiv:1111.6097] [INSPIRE].

[26] CMS collaboration, Performance of electron reconstruction and selection with the CMS detector in proton-proton collisions at $\sqrt{s}=8 \mathrm{TeV}, 2015$ JINST $10 \mathrm{P} 06005$ [arXiv: 1502.02701] [INSPIRE].

[27] CMS collaboration, Performance of CMS muon reconstruction in pp collision events at $\sqrt{s}=7 \mathrm{TeV}, 2012$ JINST 7 P10002 [arXiv:1206.4071] [INSPIRE].

[28] CMS collaboration, Reconstruction and identification of $\tau$ lepton decays to hadrons and $\nu_{\tau}$ at CMS, 2016 JINST 11 P01019 [arXiv:1510.07488] [INSPIRE].

[29] CMS collaboration, Jet energy scale and resolution in the CMS experiment in pp collisions at $8 \mathrm{TeV}, 2017$ JINST $12 \mathrm{P} 02014$ [arXiv: 1607.03663] [INSPIRE]. 
[30] CMS collaboration, Identification of b-quark jets with the CMS experiment, 2013 JINST 8 P04013 [arXiv: 1211.4462] [INSPIRE].

[31] CMS collaboration, Identification of heavy-flavour jets with the CMS detector in pp collisions at $13 \mathrm{TeV}, 2018$ JINST 13 P05011 [arXiv:1712.07158] [INSPIRE].

[32] J. Alwall et al., The automated computation of tree-level and next-to-leading order differential cross sections and their matching to parton shower simulations, JHEP 07 (2014) 079 [arXiv: 1405.0301] [INSPIRE].

[33] S. Alioli, P. Nason, C. Oleari and E. Re, NLO single-top production matched with shower in POWHEG: s-and t-channel contributions, JHEP 09 (2009) 111 [Erratum ibid. 02 (2010) 011] [arXiv:0907.4076] [INSPIRE].

[34] E. Re, Single-top Wt-channel production matched with parton showers using the POWHEG method, Eur. Phys. J. C 71 (2011) 1547 [arXiv:1009.2450] [InSPIRE].

[35] NNPDF collaboration, R.D. Ball et al., Parton distributions for the LHC run II, JHEP 04 (2015) 040 [arXiv: 1410.8849] [InSPIRE].

[36] T. Sjöstrand, S. Mrenna and P.Z. Skands, PYTHIA 6.4 physics and manual, JHEP 05 (2006) 026 [hep-ph/0603175] [INSPIRE].

[37] T. Sjöstrand, S. Mrenna and P.Z. Skands, A brief introduction to PYTHIA 8.1, Comput. Phys. Commun. 178 (2008) 852 [arXiv:0710.3820] [INSPIRE].

[38] CMS collaboration, Event generator tunes obtained from underlying event and multiparton scattering measurements, Eur. Phys. J. C 76 (2016) 155 [arXiv:1512.00815] [InSPIRE].

[39] GEANT4 collaboration, S. Agostinelli et al., GEANT4: a simulation toolkit, Nucl. Instrum. Meth. A 506 (2003) 250 [inSPIRE].

[40] W. Beenakker, R. Höpker and M. Spira, PROSPINO: a program for the production of supersymmetric particles in next-to-leading order QCD, hep-ph/9611232 [INSPIRE].

[41] C. Borschensky et al., Squark and gluino production cross sections in pp collisions at $\sqrt{s}=13,14,33$ and 100 TeV, Eur. Phys. J. C 74 (2014) 3174 [arXiv:1407.5066] [InSPIRE].

[42] W. Beenakker, R. Höpker, M. Spira and P.M. Zerwas, Squark and gluino production at hadron colliders, Nucl. Phys. B 492 (1997) 51 [hep-ph/9610490] [INSPIRE].

[43] A. Kulesza and L. Motyka, Threshold resummation for squark-antisquark and gluino-pair production at the LHC, Phys. Rev. Lett. 102 (2009) 111802 [arXiv:0807.2405] [INSPIRE].

[44] A. Kulesza and L. Motyka, Soft gluon resummation for the production of gluino-gluino and squark-antisquark pairs at the LHC, Phys. Rev. D 80 (2009) 095004 [arXiv:0905.4749] [INSPIRE].

[45] W. Beenakker, S. Brensing, M. Krämer, A. Kulesza, E. Laenen and I. Niessen, Soft-gluon resummation for squark and gluino hadroproduction, JHEP 12 (2009) 041 [arXiv:0909.4418] [INSPIRE].

[46] W. Beenakker et al., Squark and gluino hadroproduction, Int. J. Mod. Phys. A 26 (2011) 2637 [arXiv: 1105.1110] [INSPIRE].

[47] CMS collaboration, S. Abdullin, P. Azzi, F. Beaudette, P. Janot and A. Perrotta, The fast simulation of the CMS detector at LHC, J. Phys. Conf. Ser. 331 (2011) 032049 [InSPIRE].

[48] CMS collaboration, CMS luminosity measurements for the 2016 data taking period, CMS-PAS-LUM-17-001, CERN, Geneva, Switzerland, (2017). 
[49] CMS collaboration, Measurement of the inelastic proton-proton cross section at $\sqrt{s}=13 \mathrm{TeV}, \mathrm{JHEP} 07$ (2018) 161 [arXiv: 1802.02613] [INSPIRE].

[50] CMS collaboration, Search for supersymmetry in multijet events with missing transverse momentum in proton-proton collisions at 13 TeV, Phys. Rev. D 96 (2017) 032003 [arXiv: 1704.07781] [INSPIRE].

[51] CMS collaboration, Search for electroweak production of charginos and neutralinos in $W H$ events in proton-proton collisions at $\sqrt{s}=13$ TeV, JHEP 11 (2017) 029 [arXiv:1706.09933] [INSPIRE].

[52] A. Kalogeropoulos and J. Alwall, The SysCalc code: a tool to derive theoretical systematic uncertainties, arXiv:1801.08401 [INSPIRE].

[53] L. Rokach and O. Maimon, Data mining with decision trees: theory and applications, World Scientific Pub Co Inc., Singapore, (2008) [ISBN:978-981-277-171-1].

[54] A. Höcker et al., TMVA - toolkit for multivariate data analysis, PoS (ACAT) 040 [physics/0703039] [INSPIRE].

[55] G. Cowan, K. Cranmer, E. Gross and O. Vitells, Asymptotic formulae for likelihood-based tests of new physics, Eur. Phys. J. C 71 (2011) 1554 [Erratum ibid. C 73 (2013) 2501] [arXiv: 1007.1727] [INSPIRE].

[56] T. Junk, Confidence level computation for combining searches with small statistics, Nucl. Instrum. Meth. A 434 (1999) 435 [hep-ex/9902006] [INSPIRE].

[57] A.L. Read, Presentation of search results: the $C L_{s}$ technique, J. Phys. G 28 (2002) 2693 [INSPIRE].

[58] ATLAS, CMS and LHC Higgs Combination Group collaborations, Procedure for the LHC Higgs boson search combination in Summer 2011, CMS-NOTE-2011-005, CERN, Geneva, Switzerland, (2011) [ATL-PHYS-PUB-2011-011]. 


\section{The CMS collaboration}

\section{Yerevan Physics Institute, Yerevan, Armenia}

A.M. Sirunyan, A. Tumasyan

\section{Institut für Hochenergiephysik, Wien, Austria}

W. Adam, F. Ambrogi, E. Asilar, T. Bergauer, J. Brandstetter, E. Brondolin, M. Dragicevic, J. Erö, A. Escalante Del Valle, M. Flechl, R. Frühwirth ${ }^{1}$, V.M. Ghete, J. Hrubec, M. Jeitler ${ }^{1}$, N. Krammer, I. Krätschmer, D. Liko, T. Madlener, I. Mikulec, N. Rad, H. Rohringer, J. Schieck ${ }^{1}$, R. Schöfbeck, M. Spanring, D. Spitzbart, A. Taurok, W. Waltenberger, J. Wittmann, C.-E. Wulz ${ }^{1}$, M. Zarucki

Institute for Nuclear Problems, Minsk, Belarus

V. Chekhovsky, V. Mossolov, J. Suarez Gonzalez

Universiteit Antwerpen, Antwerpen, Belgium

E.A. De Wolf, D. Di Croce, X. Janssen, J. Lauwers, M. Pieters, M. Van De Klundert, H. Van Haevermaet, P. Van Mechelen, N. Van Remortel

\section{Vrije Universiteit Brussel, Brussel, Belgium}

S. Abu Zeid, F. Blekman, J. D'Hondt, I. De Bruyn, J. De Clercq, K. Deroover, G. Flouris, D. Lontkovskyi, S. Lowette, I. Marchesini, S. Moortgat, L. Moreels, Q. Python, K. Skovpen, S. Tavernier, W. Van Doninck, P. Van Mulders, I. Van Parijs

Université Libre de Bruxelles, Bruxelles, Belgium

D. Beghin, B. Bilin, H. Brun, B. Clerbaux, G. De Lentdecker, H. Delannoy, B. Dorney, G. Fasanella, L. Favart, R. Goldouzian, A. Grebenyuk, A.K. Kalsi, T. Lenzi, J. Luetic, N. Postiau, E. Starling, L. Thomas, C. Vander Velde, P. Vanlaer, D. Vannerom, Q. Wang

\section{Ghent University, Ghent, Belgium}

T. Cornelis, D. Dobur, A. Fagot, M. Gul, I. Khvastunov², D. Poyraz, C. Roskas, D. Trocino, M. Tytgat, W. Verbeke, B. Vermassen, M. Vit, N. Zaganidis

\section{Université Catholique de Louvain, Louvain-la-Neuve, Belgium}

H. Bakhshiansohi, O. Bondu, S. Brochet, G. Bruno, C. Caputo, P. David, C. Delaere, M. Delcourt, B. Francois, A. Giammanco, G. Krintiras, V. Lemaitre, A. Magitteri, A. Mertens, M. Musich, K. Piotrzkowski, A. Saggio, M. Vidal Marono, S. Wertz, J. Zobec

\section{Centro Brasileiro de Pesquisas Fisicas, Rio de Janeiro, Brazil}

F.L. Alves, G.A. Alves, L. Brito, G. Correia Silva, C. Hensel, A. Moraes, M.E. Pol, P. Rebello Teles

Universidade do Estado do Rio de Janeiro, Rio de Janeiro, Brazil

E. Belchior Batista Das Chagas, W. Carvalho, J. Chinellato ${ }^{3}$, E. Coelho, E.M. Da Costa, G.G. Da Silveira ${ }^{4}$, D. De Jesus Damiao, C. De Oliveira Martins, S. Fonseca De Souza, H. Malbouisson, D. Matos Figueiredo, M. Melo De Almeida, C. Mora Herrera, L. Mundim, H. Nogima, W.L. Prado Da Silva, L.J. Sanchez Rosas, A. Santoro, A. Sznajder, M. Thiel, E.J. Tonelli Manganote ${ }^{3}$, F. Torres Da Silva De Araujo, A. Vilela Pereira 
Universidade Estadual Paulista ${ }^{a}$, Universidade Federal do ABC ${ }^{b}$, São Paulo, Brazil

S. Ahuja ${ }^{a}$, C.A. Bernardes ${ }^{a}$, L. Calligaris $^{a}$, T.R. Fernandez Perez Tomei ${ }^{a}$, E.M. Gregores $^{b}{ }$ P.G. Mercadante ${ }^{b}$, S.F. Novaes ${ }^{a}$, SandraS. Padula ${ }^{a}$, D. Romero Abad $^{b}$

Institute for Nuclear Research and Nuclear Energy, Bulgarian Academy of Sciences, Sofia, Bulgaria

A. Aleksandrov, R. Hadjiiska, P. Iaydjiev, A. Marinov, M. Misheva, M. Rodozov,

M. Shopova, G. Sultanov

University of Sofia, Sofia, Bulgaria

A. Dimitrov, L. Litov, B. Pavlov, P. Petkov

Beihang University, Beijing, China

W. Fang ${ }^{5}$, X. Gao ${ }^{5}$, L. Yuan

Institute of High Energy Physics, Beijing, China

M. Ahmad, J.G. Bian, G.M. Chen, H.S. Chen, M. Chen, Y. Chen, C.H. Jiang, D. Leggat,

H. Liao, Z. Liu, F. Romeo, S.M. Shaheen, A. Spiezia, J. Tao, C. Wang, Z. Wang, E. Yazgan,

H. Zhang, J. Zhao

State Key Laboratory of Nuclear Physics and Technology, Peking University, Beijing, China

Y. Ban, G. Chen, J. Li, L. Li, Q. Li, Y. Mao, S.J. Qian, D. Wang, Z. Xu

Tsinghua University, Beijing, China

Y. Wang

Universidad de Los Andes, Bogota, Colombia

C. Avila, A. Cabrera, C.A. Carrillo Montoya, L.F. Chaparro Sierra, C. Florez,

C.F. González Hernández, M.A. Segura Delgado

University of Split, Faculty of Electrical Engineering, Mechanical Engineering and Naval Architecture, Split, Croatia

B. Courbon, N. Godinovic, D. Lelas, I. Puljak, T. Sculac

University of Split, Faculty of Science, Split, Croatia

Z. Antunovic, M. Kovac

Institute Rudjer Boskovic, Zagreb, Croatia

V. Brigljevic, D. Ferencek, K. Kadija, B. Mesic, A. Starodumov ${ }^{6}$, T. Susa

University of Cyprus, Nicosia, Cyprus

M.W. Ather, A. Attikis, G. Mavromanolakis, J. Mousa, C. Nicolaou, F. Ptochos, P.A. Razis, H. Rykaczewski

Charles University, Prague, Czech Republic

M. Finger ${ }^{7}$, M. Finger Jr. ${ }^{7}$ 
Escuela Politecnica Nacional, Quito, Ecuador

E. Ayala

Universidad San Francisco de Quito, Quito, Ecuador

E. Carrera Jarrin

Academy of Scientific Research and Technology of the Arab Republic of Egypt, Egyptian Network of High Energy Physics, Cairo, Egypt

S. Elgammal ${ }^{8}$, S. Khalil ${ }^{9}$, A. Mahrous ${ }^{10}$

National Institute of Chemical Physics and Biophysics, Tallinn, Estonia

S. Bhowmik, A. Carvalho Antunes De Oliveira, R.K. Dewanjee, K. Ehataht, M. Kadastik, M. Raidal, C. Veelken

Department of Physics, University of Helsinki, Helsinki, Finland

P. Eerola, H. Kirschenmann, J. Pekkanen, M. Voutilainen

Helsinki Institute of Physics, Helsinki, Finland

J. Havukainen, J.K. Heikkilä, T. Järvinen, V. Karimäki, R. Kinnunen, T. Lampén, K. Lassila-Perini, S. Laurila, S. Lehti, T. Lindén, P. Luukka, T. Mäenpää, H. Siikonen, E. Tuominen, J. Tuominiemi

Lappeenranta University of Technology, Lappeenranta, Finland

T. Tuuva

IRFU, CEA, Université Paris-Saclay, Gif-sur-Yvette, France

M. Besancon, F. Couderc, M. Dejardin, D. Denegri, J.L. Faure, F. Ferri, S. Ganjour, A. Givernaud, P. Gras, G. Hamel de Monchenault, P. Jarry, C. Leloup, E. Locci, J. Malcles, G. Negro, J. Rander, A. Rosowsky, M.Ö. Sahin, M. Titov

Laboratoire Leprince-Ringuet, Ecole polytechnique, CNRS/IN2P3, Université Paris-Saclay, Palaiseau, France

A. Abdulsalam ${ }^{11}$, C. Amendola, I. Antropov, F. Beaudette, P. Busson, C. Charlot, R. Granier de Cassagnac, I. Kucher, S. Lisniak, A. Lobanov, J. Martin Blanco, M. Nguyen, C. Ochando, G. Ortona, P. Pigard, R. Salerno, J.B. Sauvan, Y. Sirois, A.G. Stahl Leiton, A. Zabi, A. Zghiche

Université de Strasbourg, CNRS, IPHC UMR 7178, F-67000 Strasbourg, France

J.-L. Agram ${ }^{12}$, J. Andrea, D. Bloch, J.-M. Brom, E.C. Chabert, V. Cherepanov, C. Collard, E. Conte ${ }^{12}$, J.-C. Fontaine ${ }^{12}$, D. Gelé, U. Goerlach, M. Jansová, A.-C. Le Bihan, N. Tonon, P. Van Hove

Centre de Calcul de l'Institut National de Physique Nucleaire et de Physique des Particules, CNRS/IN2P3, Villeurbanne, France

S. Gadrat 
Université de Lyon, Université Claude Bernard Lyon 1, CNRS-IN2P3, Institut de Physique Nucléaire de Lyon, Villeurbanne, France

S. Beauceron, C. Bernet, G. Boudoul, N. Chanon, R. Chierici, D. Contardo, P. Depasse, H. El Mamouni, J. Fay, L. Finco, S. Gascon, M. Gouzevitch, G. Grenier, B. Ille, F. Lagarde, I.B. Laktineh, H. Lattaud, M. Lethuillier, L. Mirabito, A.L. Pequegnot, S. Perries, A. Popov ${ }^{13}$, V. Sordini, M. Vander Donckt, S. Viret, S. Zhang

Georgian Technical University, Tbilisi, Georgia

A. Khvedelidze ${ }^{7}$

Tbilisi State University, Tbilisi, Georgia

Z. Tsamalaidze ${ }^{7}$

RWTH Aachen University, I. Physikalisches Institut, Aachen, Germany

C. Autermann, L. Feld, M.K. Kiesel, K. Klein, M. Lipinski, M. Preuten, M.P. Rauch, C. Schomakers, J. Schulz, M. Teroerde, B. Wittmer, V. Zhukov ${ }^{13}$

RWTH Aachen University, III. Physikalisches Institut A, Aachen, Germany

A. Albert, D. Duchardt, M. Endres, M. Erdmann, T. Esch, R. Fischer, S. Ghosh, A. Güth, T. Hebbeker, C. Heidemann, K. Hoepfner, H. Keller, S. Knutzen, L. Mastrolorenzo, M. Merschmeyer, A. Meyer, P. Millet, S. Mukherjee, T. Pook, M. Radziej, H. Reithler, M. Rieger, F. Scheuch, A. Schmidt, D. Teyssier

RWTH Aachen University, III. Physikalisches Institut B, Aachen, Germany

G. Flügge, O. Hlushchenko, B. Kargoll, T. Kress, A. Künsken, T. Müller, A. Nehrkorn, A. Nowack, C. Pistone, O. Pooth, H. Sert, A. Stahl ${ }^{14}$

\section{Deutsches Elektronen-Synchrotron, Hamburg, Germany}

M. Aldaya Martin, T. Arndt, C. Asawatangtrakuldee, I. Babounikau, K. Beernaert, O. Behnke, U. Behrens, A. Bermúdez Martínez, D. Bertsche, A.A. Bin Anuar, K. Borras ${ }^{15}$, V. Botta, A. Campbell, P. Connor, C. Contreras-Campana, F. Costanza, V. Danilov, A. De Wit, M.M. Defranchis, C. Diez Pardos, D. Domínguez Damiani, G. Eckerlin, T. Eichhorn, A. Elwood, E. Eren, E. Gallo ${ }^{16}$, A. Geiser, J.M. Grados Luyando, A. Grohsjean, P. Gunnellini, M. Guthoff, A. Harb, J. Hauk, H. Jung, M. Kasemann, J. Keaveney, C. Kleinwort, J. Knolle, D. Krücker, W. Lange, A. Lelek, T. Lenz, K. Lipka, W. Lohmann ${ }^{17}$, R. Mankel, I.-A. Melzer-Pellmann, A.B. Meyer, M. Meyer, M. Missiroli, G. Mittag, J. Mnich, V. Myronenko, S.K. Pflitsch, D. Pitzl, A. Raspereza, M. Savitskyi, P. Saxena, P. Schütze, C. Schwanenberger, R. Shevchenko, A. Singh, N. Stefaniuk, H. Tholen, A. Vagnerini, G.P. Van Onsem, R. Walsh, Y. Wen, K. Wichmann, C. Wissing, O. Zenaiev

\section{University of Hamburg, Hamburg, Germany}

R. Aggleton, S. Bein, A. Benecke, V. Blobel, M. Centis Vignali, T. Dreyer, E. Garutti, D. Gonzalez, J. Haller, A. Hinzmann, M. Hoffmann, A. Karavdina, G. Kasieczka, R. Klanner, R. Kogler, N. Kovalchuk, S. Kurz, V. Kutzner, J. Lange, D. Marconi, J. Multhaup, M. Niedziela, D. Nowatschin, A. Perieanu, A. Reimers, O. Rieger, C. Scharf, P. Schleper, S. Schumann, J. Schwandt, J. Sonneveld, H. Stadie, G. Steinbrück, F.M. Stober, M. Stöver, D. Troendle, E. Usai, A. Vanhoefer, B. Vormwald 
Institut für Experimentelle Teilchenphysik, Karlsruhe, Germany

M. Akbiyik, C. Barth, M. Baselga, S. Baur, E. Butz, R. Caspart, T. Chwalek, F. Colombo,

W. De Boer, A. Dierlamm, N. Faltermann, B. Freund, M. Giffels, M.A. Harrendorf, F. Hartmann ${ }^{14}$, S.M. Heindl, U. Husemann, F. Kassel ${ }^{14}$, I. Katkov ${ }^{13}$, S. Kudella, H. Mildner, S. Mitra, M.U. Mozer, Th. Müller, M. Plagge, G. Quast, K. Rabbertz, M. Schröder, I. Shvetsov, G. Sieber, H.J. Simonis, R. Ulrich, S. Wayand, M. Weber, T. Weiler, S. Williamson, C. Wöhrmann, R. Wolf

Institute of Nuclear and Particle Physics (INPP), NCSR Demokritos, Aghia Paraskevi, Greece

G. Anagnostou, G. Daskalakis, T. Geralis, A. Kyriakis, D. Loukas, G. Paspalaki, I. TopsisGiotis

National and Kapodistrian University of Athens, Athens, Greece

G. Karathanasis, S. Kesisoglou, P. Kontaxakis, A. Panagiotou, N. Saoulidou, E. Tziaferi, K. Vellidis

National Technical University of Athens, Athens, Greece

K. Kousouris, I. Papakrivopoulos, G. Tsipolitis

University of Ioánnina, Ioánnina, Greece

I. Evangelou, C. Foudas, P. Gianneios, P. Katsoulis, P. Kokkas, S. Mallios, N. Manthos, I. Papadopoulos, E. Paradas, J. Strologas, F.A. Triantis, D. Tsitsonis

MTA-ELTE Lendület CMS Particle and Nuclear Physics Group, Eötvös Loránd University, Budapest, Hungary

M. Csanad, N. Filipovic, P. Major, M.I. Nagy, G. Pasztor, O. Surányi, G.I. Veres

Wigner Research Centre for Physics, Budapest, Hungary

G. Bencze, C. Hajdu, D. Horvath ${ }^{18}$, Á. Hunyadi, F. Sikler, T.Á. Vámi, V. Veszpremi, G. Vesztergombi ${ }^{\dagger}$

Institute of Nuclear Research ATOMKI, Debrecen, Hungary

N. Beni, S. Czellar, J. Karancsi20, A. Makovec, J. Molnar, Z. Szillasi

Institute of Physics, University of Debrecen, Debrecen, Hungary

M. Bartók ${ }^{19}$, P. Raics, Z.L. Trocsanyi, B. Ujvari

Indian Institute of Science (IISc), Bangalore, India

S. Choudhury, J.R. Komaragiri

National Institute of Science Education and Research, HBNI, Bhubaneswar, India

S. Bahinipati2 ${ }^{21}$, P. Mal, K. Mandal, A. Nayak ${ }^{22}$, D.K. Sahoo ${ }^{21}$, S.K. Swain

Panjab University, Chandigarh, India

S. Bansal, S.B. Beri, V. Bhatnagar, S. Chauhan, R. Chawla, N. Dhingra, R. Gupta,

A. Kaur, A. Kaur, M. Kaur, S. Kaur, R. Kumar, P. Kumari, M. Lohan, A. Mehta, K. Sandeep, S. Sharma, J.B. Singh, G. Walia 
University of Delhi, Delhi, India

A. Bhardwaj, B.C. Choudhary, R.B. Garg, M. Gola, S. Keshri, Ashok Kumar, S. Malhotra, M. Naimuddin, P. Priyanka, K. Ranjan, Aashaq Shah, R. Sharma

Saha Institute of Nuclear Physics, HBNI, Kolkata, India

R. Bhardwaj ${ }^{23}$, M. Bharti, R. Bhattacharya, S. Bhattacharya, U. Bhawandeep ${ }^{23}$, D. Bhowmik, S. Dey, S. Dutt ${ }^{23}$, S. Dutta, S. Ghosh, K. Mondal, S. Nandan, A. Purohit, P.K. Rout, A. Roy, S. Roy Chowdhury, S. Sarkar, M. Sharan, B. Singh, S. Thakur ${ }^{23}$

Indian Institute of Technology Madras, Madras, India

P.K. Behera

Bhabha Atomic Research Centre, Mumbai, India

R. Chudasama, D. Dutta, V. Jha, V. Kumar, P.K. Netrakanti, L.M. Pant, P. Shukla

Tata Institute of Fundamental Research-A, Mumbai, India

T. Aziz, M.A. Bhat, S. Dugad, B. Mahakud, G.B. Mohanty, N. Sur, B. Sutar, RavindraKumar Verma

Tata Institute of Fundamental Research-B, Mumbai, India

S. Banerjee, S. Bhattacharya, S. Chatterjee, P. Das, M. Guchait, Sa. Jain, S. Kumar, M. Maity ${ }^{24}$, G. Majumder, K. Mazumdar, N. Sahoo, T. Sarkar ${ }^{24}$

Indian Institute of Science Education and Research (IISER), Pune, India

S. Chauhan, S. Dube, V. Hegde, A. Kapoor, K. Kothekar, S. Pandey, A. Rane, S. Sharma

Institute for Research in Fundamental Sciences (IPM), Tehran, Iran

S. Chenarani ${ }^{25}$, E. Eskandari Tadavani, S.M. Etesami ${ }^{25}$, M. Khakzad, M. Mohammadi Najafabadi, M. Naseri, F. Rezaei Hosseinabadi, B. Safarzadeh ${ }^{26}$, M. Zeinali

University College Dublin, Dublin, Ireland

M. Felcini, M. Grunewald

INFN Sezione di Bari ${ }^{a}$, Università di Bari ${ }^{b}$, Politecnico di Bari ${ }^{c}$, Bari, Italy M. Abbrescia ${ }^{a, b}$, C. Calabria ${ }^{a, b}$, A. Colaleo ${ }^{a}$, D. Creanza ${ }^{a, c}$, L. Cristella ${ }^{a, b}$,

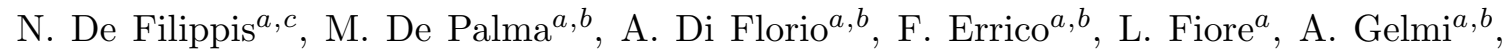

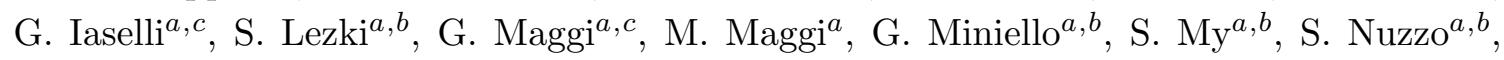
A. Pompili ${ }^{a, b}$, G. Pugliese ${ }^{a, c}$, R. Radogna ${ }^{a}$, A. Ranieri ${ }^{a}$, G. Selvaggi ${ }^{a, b}$, A. Sharma ${ }^{a}$, L. Silvestris ${ }^{a}, 14$, R. Venditti ${ }^{a}$, P. Verwilligen $^{a}$, G. Zito ${ }^{a}$

INFN Sezione di Bologna ${ }^{a}$, Università di Bologna ${ }^{b}$, Bologna, Italy

G. Abbiendi ${ }^{a}$, C. Battilana ${ }^{a, b}$, D. Bonacorsi ${ }^{a}, b$, L. Borgonovi $^{a}, b$, S. Braibant-Giacomelli ${ }^{a, b}$, L. Brigliadoria ${ }^{a, b}$, R. Campanini ${ }^{a, b}$, P. Capiluppi ${ }^{a, b}$, A. Castro ${ }^{a, b}$, F.R. Cavallo ${ }^{a}$, S.S. Chhibra ${ }^{a, b}$, G. Codispoti ${ }^{a, b}$, M. Cuffiani ${ }^{a, b}$, G.M. Dallavalle ${ }^{a}$, F. Fabbri ${ }^{a}$, A. Fanfani ${ }^{a, b}$, P. Giacomelli ${ }^{a}$, C. Grandi ${ }^{a}$, L. Guiducci ${ }^{a}, b$, S. Marcellini ${ }^{a}$, G. Masetti ${ }^{a}$, A. Montanari ${ }^{a}$,

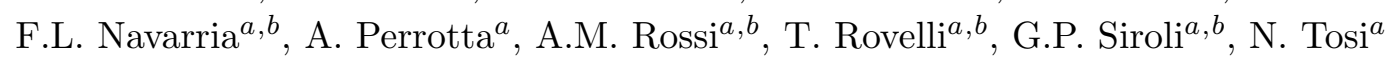

INFN Sezione di Catania ${ }^{a}$, Università di Catania ${ }^{b}$, Catania, Italy

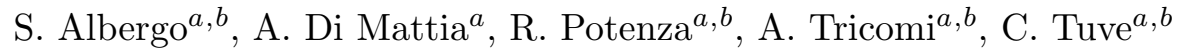


INFN Sezione di Firenze ${ }^{a}$, Università di Firenze ${ }^{b}$, Firenze, Italy

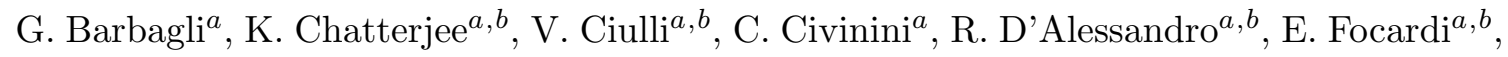

G. Latino, P. Lenzi ${ }^{a}, b$, M. Meschini ${ }^{a}$, S. Paoletti ${ }^{a}$, L. Russo $^{a, 27}$, G. Sguazzoni $^{a}$, D. Strom ${ }^{a}$,

L. Viliani ${ }^{a}$

INFN Laboratori Nazionali di Frascati, Frascati, Italy

L. Benussi, S. Bianco, F. Fabbri, D. Piccolo, F. Primavera ${ }^{14}$

INFN Sezione di Genova ${ }^{a}$, Università di Genova ${ }^{b}$, Genova, Italy

F. Ferro ${ }^{a}$, F. Ravera ${ }^{a, b}$, E. Robutti $^{a}$, S. Tosi ${ }^{a, b}$

INFN Sezione di Milano-Bicocca ${ }^{a}$, Università di Milano-Bicocca ${ }^{b}$, Milano, Italy

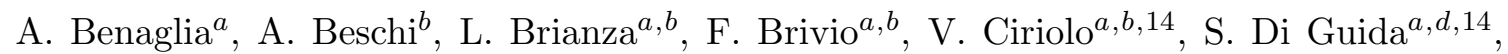
M.E. Dinardo ${ }^{a, b}$, S. Fiorendi ${ }^{a}, b$, S. Gennai ${ }^{a}$, A. Ghezzi ${ }^{a, b}$, P. Govoni $^{a}{ }^{a}$, M. Malberti $^{a, b}$,

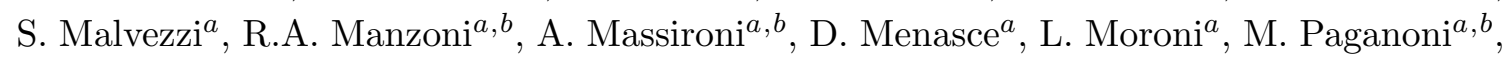
D. Pedrini ${ }^{a}$, S. Ragazzi ${ }^{a, b}$, T. Tabarelli de Fatis ${ }^{a, b}$

INFN Sezione di Napoli ${ }^{a}$, Università di Napoli 'Federico II' ${ }^{b}$, Napoli, Italy, Università della Basilicata ${ }^{c}$, Potenza, Italy, Università G. Marconi ${ }^{d}$, Roma, Italy

S. Buontempo ${ }^{a}$, N. Cavallo ${ }^{a, c}$, A. Di Crescenzo ${ }^{a, b}$, F. Fabozzi $^{a, c}$, F. Fienga $^{a, b}$, G. Galati $^{a, b}$, A.O.M. Iorio ${ }^{a, b}$, W.A. Khan ${ }^{a}$, L. Lista ${ }^{a}$, S. Meola ${ }^{a, d, 14}$, P. Paolucci ${ }^{a, 14}$, C. Sciacca ${ }^{a, b}$, E. Voevodina ${ }^{a, b}$

INFN Sezione di Padova ${ }^{a}$, Università di Padova ${ }^{b}$, Padova, Italy, Università di Trento ${ }^{c}$, Trento, Italy

P. Azzi $^{a}$, N. Bacchetta ${ }^{a}$, L. Benato ${ }^{a, b}$, D. Bisello ${ }^{a, b}$, A. Boletti ${ }^{a, b}$, A. Bragagnolo, R. Carlin ${ }^{a, b}$, P. Checchia ${ }^{a}$, M. Dall'Osso ${ }^{a, b}$, P. De Castro Manzano ${ }^{a}$, T. Dorigo ${ }^{a}$, U. Gasparini $^{a, b}$, A. Gozzelino $^{a}$, S. Lacaprara ${ }^{a}$, P. Lujan, M. Margoni ${ }^{a, b}$, A.T. Meneguzzo $^{a, b}$, F. Montecassiano ${ }^{a}$, N. Pozzobon ${ }^{a, b}$, P. Ronchese ${ }^{a, b}$, R. Rossin ${ }^{a, b}$, F. Simonetto ${ }^{a, b}$, A. Tiko, E. Torassa ${ }^{a}$, M. Zanetti ${ }^{a, b}$, P. Zotto ${ }^{a, b}$, G. Zumerle ${ }^{a, b}$

INFN Sezione di Pavia ${ }^{a}$, Università di Pavia ${ }^{b}$, Pavia, Italy

A. Braghieri ${ }^{a}$, A. Magnani ${ }^{a}$, P. Montagna ${ }^{a, b}$, S.P. Ratti ${ }^{a}, b$, V. $\operatorname{Re}^{a}$, M. Ressegotti ${ }^{a, b}$, C. Riccardi ${ }^{a, b}$, P. Salvini ${ }^{a}$, I. Vai ${ }^{a, b}$, P. Vitulo ${ }^{a, b}$

INFN Sezione di Perugia ${ }^{a}$, Università di Perugia ${ }^{b}$, Perugia, Italy

L. Alunni Solestizi ${ }^{a}, b$, M. Biasini ${ }^{a, b}$, G.M. Bilei ${ }^{a}$, C. Cecchi ${ }^{a, b}$, D. Ciangottini ${ }^{a, b}$, L. Fanò $\grave{o}^{a, b}$, P. Lariccia ${ }^{a, b}$, E. Manoni ${ }^{a}$, G. Mantovani ${ }^{a, b}$, V. Mariani ${ }^{a, b}$, M. Menichelli ${ }^{a}$, A. Rossi ${ }^{a, b}$, A. Santocchia ${ }^{a, b}$, D. Spiga ${ }^{a}$

INFN Sezione di Pisa ${ }^{a}$, Università di Pisa ${ }^{b}$, Scuola Normale Superiore di Pisa ${ }^{c}$, Pisa, Italy

K. Androsov ${ }^{a}$, P. Azzurri ${ }^{a}$, G. Bagliesi ${ }^{a}$, L. Bianchini ${ }^{a}$, T. Boccali ${ }^{a}$, L. Borrello, R. Castaldi ${ }^{a}$, M.A. Ciocci ${ }^{a}, b$, R. Dell'Orso ${ }^{a}$, G. Fedi ${ }^{a}$, L. Giannini ${ }^{a, c}$, A. Giassi ${ }^{a}$, M.T. Grippo ${ }^{a}$, F. Ligabue ${ }^{a, c}$, E. Manca ${ }^{a, c}$, G. Mandorli ${ }^{a, c}$, A. Messineo ${ }^{a, b}$, F. Palla ${ }^{a}$, A. Rizzi ${ }^{a, b}$, P. Spagnolo ${ }^{a}$, R. Tenchini ${ }^{a}$, G. Tonelli ${ }^{a}, b$, A. Venturi ${ }^{a}$, P.G. Verdini ${ }^{a}$ 
INFN Sezione di Roma ${ }^{a}$, Sapienza Università di Roma ${ }^{b}$, Rome, Italy

L. Barone ${ }^{a, b}$, F. Cavallari ${ }^{a}$, M. Cipriani ${ }^{a, b}$, N. Daci ${ }^{a}$, D. Del Re ${ }^{a, b}$, E. Di Marco ${ }^{a, b}$, M. Diemoz ${ }^{a}$, S. Gelli ${ }^{a, b}$, E. Longo ${ }^{a}, b$, B. Marzocchi ${ }^{a}, b$, P. Meridiani ${ }^{a}$, G. Organtini ${ }^{a, b}$,

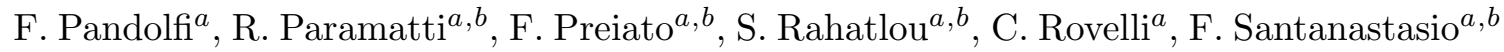

INFN Sezione di Torino ${ }^{a}$, Università di Torino ${ }^{b}$, Torino, Italy, Università del Piemonte Orientale ${ }^{c}$, Novara, Italy

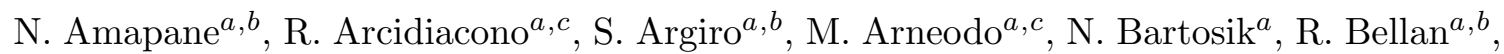

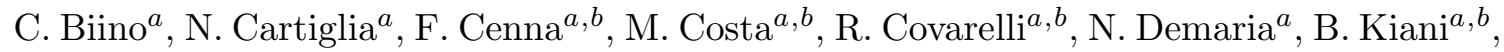
C. Mariotti $^{a}$, S. Maselli ${ }^{a}$, E. Migliore ${ }^{a, b}$, V. Monaco ${ }^{a, b}$, E. Monteil ${ }^{a, b}$, M. Monteno ${ }^{a}$, M.M. Obertino ${ }^{a, b}$, L. Pacher ${ }^{a, b}$, N. Pastrone ${ }^{a}$, M. Pelliccioni $^{a}$, G.L. Pinna Angioni ${ }^{a}, b$, A. Romero ${ }^{a, b}$, M. Ruspa ${ }^{a, c}$, R. Sacchi ${ }^{a, b}$, K. Shchelina ${ }^{a, b}$, V. Sola ${ }^{a}$, A. Solano ${ }^{a, b}$, A. Staiano ${ }^{a}$

INFN Sezione di Trieste ${ }^{a}$, Università di Trieste ${ }^{b}$, Trieste, Italy

S. Belforte ${ }^{a}$, V. Candelise ${ }^{a, b}$, M. Casarsa ${ }^{a}$, F. Cossutti ${ }^{a}$, G. Della Ricca ${ }^{a, b}$, F. Vazzoler ${ }^{a}, b$, A. Zanetti ${ }^{a}$

\section{Kyungpook National University}

D.H. Kim, G.N. Kim, M.S. Kim, J. Lee, S. Lee, S.W. Lee, C.S. Moon, Y.D. Oh, S. Sekmen, D.C. Son, Y.C. Yang

Chonnam National University, Institute for Universe and Elementary Particles, Kwangju, Korea

H. Kim, D.H. Moon, G. Oh

Hanyang University, Seoul, Korea

J. Goh, T.J. Kim

Korea University, Seoul, Korea

S. Cho, S. Choi, Y. Go, D. Gyun, S. Ha, B. Hong, Y. Jo, K. Lee, K.S. Lee, S. Lee, J. Lim, S.K. Park, Y. Roh

Sejong University, Seoul, Korea

H.S. Kim

\section{Seoul National University, Seoul, Korea}

J. Almond, J. Kim, J.S. Kim, H. Lee, K. Lee, K. Nam, S.B. Oh, B.C. Radburn-Smith, S.h. Seo, U.K. Yang, H.D. Yoo, G.B. Yu

University of Seoul, Seoul, Korea

H. Kim, J.H. Kim, J.S.H. Lee, I.C. Park

Sungkyunkwan University, Suwon, Korea

Y. Choi, C. Hwang, J. Lee, I. Yu

Vilnius University, Vilnius, Lithuania

V. Dudenas, A. Juodagalvis, J. Vaitkus 
National Centre for Particle Physics, Universiti Malaya, Kuala Lumpur, Malaysia

I. Ahmed, Z.A. Ibrahim, M.A.B. Md Ali ${ }^{28}$, F. Mohamad Idris ${ }^{29}$, W.A.T. Wan Abdullah, M.N. Yusli, Z. Zolkapli

Centro de Investigacion y de Estudios Avanzados del IPN, Mexico City, Mexico M.C. Duran-Osuna, H. Castilla-Valdez, E. De La Cruz-Burelo, G. Ramirez-Sanchez, I. Heredia-De La Cruz ${ }^{30}$, R.I. Rabadan-Trejo, R. Lopez-Fernandez, J. Mejia Guisao, R Reyes-Almanza, A. Sanchez-Hernandez

Universidad Iberoamericana, Mexico City, Mexico

S. Carrillo Moreno, C. Oropeza Barrera, F. Vazquez Valencia

Benemerita Universidad Autonoma de Puebla, Puebla, Mexico

J. Eysermans, I. Pedraza, H.A. Salazar Ibarguen, C. Uribe Estrada

Universidad Autónoma de San Luis Potosí, San Luis Potosí, Mexico

A. Morelos Pineda

University of Auckland, Auckland, New Zealand

D. Krofcheck

University of Canterbury, Christchurch, New Zealand

S. Bheesette, P.H. Butler

National Centre for Physics, Quaid-I-Azam University, Islamabad, Pakistan

A. Ahmad, M. Ahmad, M.I. Asghar, Q. Hassan, H.R. Hoorani, A. Saddique, M.A. Shah, M. Shoaib, M. Waqas

National Centre for Nuclear Research, Swierk, Poland

H. Bialkowska, M. Bluj, B. Boimska, T. Frueboes, M. Górski, M. Kazana, K. Nawrocki, M. Szleper, P. Traczyk, P. Zalewski

Institute of Experimental Physics, Faculty of Physics, University of Warsaw, Warsaw, Poland

K. Bunkowski, A. Byszuk ${ }^{31}$, K. Doroba, A. Kalinowski, M. Konecki, J. Krolikowski, M. Misiura, M. Olszewski, A. Pyskir, M. Walczak

Laboratório de Instrumentação e Física Experimental de Partículas, Lisboa, Portugal

P. Bargassa, C. Beirão Da Cruz E Silva, A. Di Francesco, P. Faccioli, B. Galinhas, M. Gallinaro, J. Hollar, N. Leonardo, L. Lloret Iglesias, M.V. Nemallapudi, J. Seixas, G. Strong, O. Toldaiev, D. Vadruccio, J. Varela

Joint Institute for Nuclear Research, Dubna, Russia

S. Afanasiev, V. Alexakhin, P. Bunin, M. Gavrilenko, A. Golunov, I. Golutvin, N. Gorbounov, V. Karjavin, A. Lanev, A. Malakhov, V. Matveev ${ }^{32,33}$, P. Moisenz, V. Palichik, V. Perelygin, M. Savina, S. Shmatov, V. Smirnov, N. Voytishin, A. Zarubin 
Petersburg Nuclear Physics Institute, Gatchina (St. Petersburg), Russia

V. Golovtsov, Y. Ivanov, V. Kim ${ }^{34}$, E. Kuznetsova ${ }^{35}$, P. Levchenko, V. Murzin, V. Oreshkin, I. Smirnov, D. Sosnov, V. Sulimov, L. Uvarov, S. Vavilov, A. Vorobyev

Institute for Nuclear Research, Moscow, Russia

Yu. Andreev, A. Dermenev, S. Gninenko, N. Golubev, A. Karneyeu, M. Kirsanov, N. Krasnikov, A. Pashenkov, D. Tlisov, A. Toropin

Institute for Theoretical and Experimental Physics, Moscow, Russia

V. Epshteyn, V. Gavrilov, N. Lychkovskaya, V. Popov, I. Pozdnyakov, G. Safronov, A. Spiridonov, A. Stepennov, V. Stolin, M. Toms, E. Vlasov, A. Zhokin

Moscow Institute of Physics and Technology, Moscow, Russia

T. Aushev, A. Bylinkin 33

National Research Nuclear University 'Moscow Engineering Physics Institute' (MEPhI), Moscow, Russia

R. Chistov ${ }^{36}$, P. Parygin, D. Philippov, S. Polikarpov ${ }^{36}$, E. Tarkovskii

P.N. Lebedev Physical Institute, Moscow, Russia

V. Andreev, M. Azarkin ${ }^{33}$, I. Dremin ${ }^{33}$, M. Kirakosyan ${ }^{33}$, S.V. Rusakov, A. Terkulov

Skobeltsyn Institute of Nuclear Physics, Lomonosov Moscow State University, Moscow, Russia

A. Baskakov, A. Belyaev, E. Boos, V. Bunichev, M. Dubinin ${ }^{37}$, L. Dudko, A. Ershov, A. Gribushin, V. Klyukhin, O. Kodolova, I. Lokhtin, I. Miagkov, S. Obraztsov, S. Petrushanko, V. Savrin

Novosibirsk State University (NSU), Novosibirsk, Russia

V. Blinov ${ }^{38}$, T. Dimova ${ }^{38}$, L. Kardapoltsev ${ }^{38}$, D. Shtol $^{38}$, Y. Skovpen ${ }^{38}$

State Research Center of Russian Federation, Institute for High Energy Physics of NRC 'Kurchatov Institute', Protvino, Russia

I. Azhgirey, I. Bayshev, S. Bitioukov, D. Elumakhov, A. Godizov, V. Kachanov, A. Kalinin, D. Konstantinov, P. Mandrik, V. Petrov, R. Ryutin, S. Slabospitskii, A. Sobol, S. Troshin, N. Tyurin, A. Uzunian, A. Volkov

National Research Tomsk Polytechnic University, Tomsk, Russia

A. Babaev

University of Belgrade, Faculty of Physics and Vinca Institute of Nuclear Sciences, Belgrade, Serbia

P. Adzic ${ }^{39}$, P. Cirkovic, D. Devetak, M. Dordevic, J. Milosevic

Centro de Investigaciones Energéticas Medioambientales y Tecnológicas (CIEMAT), Madrid, Spain

J. Alcaraz Maestre, A. Álvarez Fernández, I. Bachiller, M. Barrio Luna, J.A. Brochero Cifuentes, M. Cerrada, N. Colino, B. De La Cruz, A. Delgado Peris, C. Fernandez Bedoya, 
J.P. Fernández Ramos, J. Flix, M.C. Fouz, O. Gonzalez Lopez, S. Goy Lopez, J.M. Hernandez, M.I. Josa, D. Moran, A. Pérez-Calero Yzquierdo, J. Puerta Pelayo, I. Redondo, L. Romero, M.S. Soares, A. Triossi

\section{Universidad Autónoma de Madrid, Madrid, Spain}

C. Albajar, J.F. de Trocóniz

\section{Universidad de Oviedo, Oviedo, Spain}

J. Cuevas, C. Erice, J. Fernandez Menendez, S. Folgueras, I. Gonzalez Caballero, J.R. González Fernández, E. Palencia Cortezon, V. Rodríguez Bouza, S. Sanchez Cruz, P. Vischia, J.M. Vizan Garcia

\section{Instituto de Física de Cantabria (IFCA), CSIC-Universidad de Cantabria,} Santander, Spain

I.J. Cabrillo, A. Calderon, B. Chazin Quero, J. Duarte Campderros, M. Fernandez, P.J. Fernández Manteca, A. García Alonso, J. Garcia-Ferrero, G. Gomez, A. Lopez Virto, J. Marco, C. Martinez Rivero, P. Martinez Ruiz del Arbol, F. Matorras, J. Piedra Gomez, C. Prieels, T. Rodrigo, A. Ruiz-Jimeno, L. Scodellaro, N. Trevisani, I. Vila, R. Vilar Cortabitarte

\section{CERN, European Organization for Nuclear Research, Geneva, Switzerland}

D. Abbaneo, B. Akgun, E. Auffray, P. Baillon, A.H. Ball, D. Barney, J. Bendavid, M. Bianco, A. Bocci, C. Botta, T. Camporesi, M. Cepeda, G. Cerminara, E. Chapon, Y. Chen, G. Cucciati, D. d'Enterria, A. Dabrowski, V. Daponte, A. David, A. De Roeck, N. Deelen, M. Dobson, T. du Pree, M. Dünser, N. Dupont, A. Elliott-Peisert, P. Everaerts, F. Fallavollita ${ }^{40}$, D. Fasanella, G. Franzoni, J. Fulcher, W. Funk, D. Gigi, A. Gilbert, K. Gill, F. Glege, D. Gulhan, J. Hegeman, V. Innocente, A. Jafari, P. Janot, O. Karacheban ${ }^{17}$, J. Kieseler, V. Knünz, A. Kornmayer, M. Krammer ${ }^{1}$, C. Lange, P. Lecoq, C. Lourenço, L. Malgeri, M. Mannelli, F. Meijers, J.A. Merlin, S. Mersi, E. Meschi, P. Milenovic ${ }^{41}$, F. Moortgat, M. Mulders, H. Neugebauer, J. Ngadiuba, S. Orfanelli, L. Orsini, F. Pantaleo ${ }^{14}$, L. Pape, E. Perez, M. Peruzzi, A. Petrilli, G. Petrucciani, A. Pfeiffer, M. Pierini, F.M. Pitters, D. Rabady, A. Racz, T. Reis, G. Rolandi ${ }^{42}$, M. Rovere, H. Sakulin, C. Schäfer, C. Schwick, M. Seidel, M. Selvaggi, A. Sharma, P. Silva, P. Sphicas ${ }^{43}$, A. Stakia, J. Steggemann, M. Tosi, D. Treille, A. Tsirou, V. Veckalns ${ }^{44}$, M. Verweij, W.D. Zeuner

\section{Paul Scherrer Institut, Villigen, Switzerland}

W. Bertl ${ }^{\dagger}$, L. Caminada ${ }^{45}$, K. Deiters, W. Erdmann, R. Horisberger, Q. Ingram, H.C. Kaestli, D. Kotlinski, U. Langenegger, T. Rohe, S.A. Wiederkehr

ETH Zurich - Institute for Particle Physics and Astrophysics (IPA), Zurich, Switzerland

M. Backhaus, L. Bäni, P. Berger, N. Chernyavskaya, G. Dissertori, M. Dittmar, M. Donegà, C. Dorfer, C. Grab, C. Heidegger, D. Hits, J. Hoss, T. Klijnsma, W. Lustermann, M. Marionneau, M.T. Meinhard, D. Meister, F. Micheli, P. Musella, F. Nessi-Tedaldi, J. Pata, F. Pauss, G. Perrin, L. Perrozzi, S. Pigazzini, M. Quittnat, M. Reichmann, D. Ruini, 
D.A. Sanz Becerra, M. Schönenberger, L. Shchutska, V.R. Tavolaro, K. Theofilatos, M.L. Vesterbacka Olsson, R. Wallny, D.H. Zhu

Universität Zürich, Zurich, Switzerland

T.K. Aarrestad, C. Amsler ${ }^{46}$, D. Brzhechko, M.F. Canelli, A. De Cosa, R. Del Burgo, S. Donato, C. Galloni, T. Hreus, B. Kilminster, I. Neutelings, D. Pinna, G. Rauco, P. Robmann, D. Salerno, K. Schweiger, C. Seitz, Y. Takahashi, A. Zucchetta

National Central University, Chung-Li, Taiwan

Y.H. Chang, K.y. Cheng, T.H. Doan, Sh. Jain, R. Khurana, C.M. Kuo, W. Lin, A. Pozdnyakov, S.S. Yu

National Taiwan University (NTU), Taipei, Taiwan

P. Chang, Y. Chao, K.F. Chen, P.H. Chen, W.-S. Hou, Arun Kumar, Y.y. Li, R.-S. Lu,

E. Paganis, A. Psallidas, A. Steen, J.f. Tsai

Chulalongkorn University, Faculty of Science, Department of Physics, Bangkok, Thailand

B. Asavapibhop, N. Srimanobhas, N. Suwonjandee

Çukurova University, Physics Department, Science and Art Faculty, Adana, Turkey

A. Bat, F. Boran, S. Cerci ${ }^{47}$, S. Damarseckin, Z.S. Demiroglu, C. Dozen, I. Dumanoglu,

S. Girgis, G. Gokbulut, Y. Guler, E. Gurpinar, I. $\mathrm{Hos}^{48}$, E.E. Kangal ${ }^{49}$, O. Kara,

A. Kayis Topaksu, U. Kiminsu, M. Oglakci, G. Onengut, K. Ozdemir ${ }^{50}$, S. Ozturk ${ }^{51}$,

D. Sunar Cerci $^{47}$, B. Tali ${ }^{47}$, U.G. Tok, S. Turkcapar, I.S. Zorbakir, C. Zorbilmez

Middle East Technical University, Physics Department, Ankara, Turkey

B. Isildak ${ }^{52}$, G. Karapinar ${ }^{53}$, M. Yalvac, M. Zeyrek

Bogazici University, Istanbul, Turkey

I.O. Atakisi, E. Gülmez, M. Kaya ${ }^{54}$, O. Kaya ${ }^{55}$, S. Tekten, E.A. Yetkin ${ }^{56}$

Istanbul Technical University, Istanbul, Turkey

M.N. Agaras, S. Atay, A. Cakir, K. Cankocak, Y. Komurcu, S. Sen ${ }^{57}$

Institute for Scintillation Materials of National Academy of Science of Ukraine, Kharkov, Ukraine

B. Grynyov

National Scientific Center, Kharkov Institute of Physics and Technology, Kharkov, Ukraine

L. Levchuk

University of Bristol, Bristol, United Kingdom

T. Alexander, F. Ball, L. Beck, J.J. Brooke, D. Burns, E. Clement, D. Cussans, O. Davignon, H. Flacher, J. Goldstein, G.P. Heath, H.F. Heath, L. Kreczko, D.M. Newbold ${ }^{58}$, S. Paramesvaran, B. Penning, T. Sakuma, D. Smith, V.J. Smith, J. Taylor 


\section{Rutherford Appleton Laboratory, Didcot, United Kingdom}

K.W. Bell, A. Belyaev ${ }^{59}$, C. Brew, R.M. Brown, D. Cieri, D.J.A. Cockerill, J.A. Coughlan, K. Harder, S. Harper, J. Linacre, E. Olaiya, D. Petyt, C.H. Shepherd-Themistocleous, A. Thea, I.R. Tomalin, T. Williams, W.J. Womersley

\section{Imperial College, London, United Kingdom}

G. Auzinger, R. Bainbridge, P. Bloch, J. Borg, S. Breeze, O. Buchmuller, A. Bundock, S. Casasso, D. Colling, L. Corpe, P. Dauncey, G. Davies, M. Della Negra, R. Di Maria, Y. Haddad, G. Hall, G. Iles, T. James, M. Komm, C. Laner, L. Lyons, A.-M. Magnan, S. Malik, A. Martelli, J. Nash ${ }^{60}$, A. Nikitenko ${ }^{6}$, V. Palladino, M. Pesaresi, A. Richards, A. Rose, E. Scott, C. Seez, A. Shtipliyski, G. Singh, M. Stoye, T. Strebler, S. Summers, A. Tapper, K. Uchida, T. Virdee ${ }^{14}$, N. Wardle, D. Winterbottom, J. Wright, S.C. Zenz

\section{Brunel University, Uxbridge, United Kingdom}

J.E. Cole, P.R. Hobson, A. Khan, P. Kyberd, C.K. Mackay, A. Morton, I.D. Reid, L. Teodorescu, S. Zahid

\section{Baylor University, Waco, U.S.A.}

K. Call, J. Dittmann, K. Hatakeyama, H. Liu, C. Madrid, B. Mcmaster, N. Pastika, C. Smith

\section{Catholic University of America, Washington DC, U.S.A.}

R. Bartek, A. Dominguez

The University of Alabama, Tuscaloosa, U.S.A.

A. Buccilli, S.I. Cooper, C. Henderson, P. Rumerio, C. West

\section{Boston University, Boston, U.S.A.}

D. Arcaro, T. Bose, D. Gastler, D. Rankin, C. Richardson, J. Rohlf, L. Sulak, D. Zou

\section{Brown University, Providence, U.S.A.}

G. Benelli, X. Coubez, D. Cutts, M. Hadley, J. Hakala, U. Heintz, J.M. Hogan ${ }^{61}$, K.H.M. Kwok, E. Laird, G. Landsberg, J. Lee, Z. Mao, M. Narain, J. Pazzini, S. Piperov, S. Sagir ${ }^{62}$, R. Syarif, D. Yu

University of California, Davis, Davis, U.S.A.

R. Band, C. Brainerd, R. Breedon, D. Burns, M. Calderon De La Barca Sanchez, M. Chertok, J. Conway, R. Conway, P.T. Cox, R. Erbacher, C. Flores, G. Funk, W. Ko, O. Kukral, R. Lander, C. Mclean, M. Mulhearn, D. Pellett, J. Pilot, S. Shalhout, M. Shi, D. Stolp, D. Taylor, K. Tos, M. Tripathi, Z. Wang, F. Zhang

\section{University of California, Los Angeles, U.S.A.}

M. Bachtis, C. Bravo, R. Cousins, A. Dasgupta, A. Florent, J. Hauser, M. Ignatenko, N. Mccoll, S. Regnard, D. Saltzberg, C. Schnaible, V. Valuev

University of California, Riverside, Riverside, U.S.A.

E. Bouvier, K. Burt, R. Clare, J.W. Gary, S.M.A. Ghiasi Shirazi, G. Hanson, G. Karapostoli, E. Kennedy, F. Lacroix, O.R. Long, M. Olmedo Negrete, M.I. Paneva, W. Si, L. Wang, H. Wei, S. Wimpenny, B.R. Yates 
University of California, San Diego, La Jolla, U.S.A.

J.G. Branson, S. Cittolin, M. Derdzinski, R. Gerosa, D. Gilbert, B. Hashemi, A. Holzner, D. Klein, G. Kole, V. Krutelyov, J. Letts, M. Masciovecchio, D. Olivito, S. Padhi, M. Pieri, M. Sani, V. Sharma, S. Simon, M. Tadel, A. Vartak, S. Wasserbaech ${ }^{63}$, J. Wood, F. Würthwein, A. Yagil, G. Zevi Della Porta

University of California, Santa Barbara - Department of Physics, Santa Barbara, U.S.A.

N. Amin, R. Bhandari, J. Bradmiller-Feld, C. Campagnari, M. Citron, A. Dishaw, V. Dutta, M. Franco Sevilla, L. Gouskos, R. Heller, J. Incandela, A. Ovcharova, H. Qu, J. Richman, D. Stuart, I. Suarez, S. Wang, J. Yoo

California Institute of Technology, Pasadena, U.S.A.

D. Anderson, A. Bornheim, J. Bunn, J.M. Lawhorn, H.B. Newman, T.Q. Nguyen, M. Spiropulu, J.R. Vlimant, R. Wilkinson, S. Xie, Z. Zhang, R.Y. Zhu

Carnegie Mellon University, Pittsburgh, U.S.A.

M.B. Andrews, T. Ferguson, T. Mudholkar, M. Paulini, M. Sun, I. Vorobiev, M. Weinberg

University of Colorado Boulder, Boulder, U.S.A.

J.P. Cumalat, W.T. Ford, F. Jensen, A. Johnson, M. Krohn, S. Leontsinis, E. MacDonald, T. Mulholland, K. Stenson, K.A. Ulmer, S.R. Wagner

Cornell University, Ithaca, U.S.A.

J. Alexander, J. Chaves, Y. Cheng, J. Chu, A. Datta, K. Mcdermott, N. Mirman, J.R. Patterson, D. Quach, A. Rinkevicius, A. Ryd, L. Skinnari, L. Soffi, S.M. Tan, Z. Tao, J. Thom, J. Tucker, P. Wittich, M. Zientek

Fermi National Accelerator Laboratory, Batavia, U.S.A.

S. Abdullin, M. Albrow, M. Alyari, G. Apollinari, A. Apresyan, A. Apyan, S. Banerjee, L.A.T. Bauerdick, A. Beretvas, J. Berryhill, P.C. Bhat, G. Bolla ${ }^{\dagger}$, K. Burkett, J.N. Butler, A. Canepa, G.B. Cerati, H.W.K. Cheung, F. Chlebana, M. Cremonesi, J. Duarte, V.D. Elvira, J. Freeman, Z. Gecse, E. Gottschalk, L. Gray, D. Green, S. Grünendahl, O. Gutsche, J. Hanlon, R.M. Harris, S. Hasegawa, J. Hirschauer, Z. Hu, B. Jayatilaka, S. Jindariani, M. Johnson, U. Joshi, B. Klima, M.J. Kortelainen, B. Kreis, S. Lammel, D. Lincoln, R. Lipton, M. Liu, T. Liu, J. Lykken, K. Maeshima, J.M. Marraffino, D. Mason, P. McBride, P. Merkel, S. Mrenna, S. Nahn, V. O'Dell, K. Pedro, C. Pena, O. Prokofyev, G. Rakness, L. Ristori, A. Savoy-Navarro ${ }^{64}$, B. Schneider, E. Sexton-Kennedy, A. Soha, W.J. Spalding, L. Spiegel, S. Stoynev, J. Strait, N. Strobbe, L. Taylor, S. Tkaczyk, N.V. Tran, L. Uplegger, E.W. Vaandering, C. Vernieri, M. Verzocchi, R. Vidal, M. Wang, H.A. Weber, A. Whitbeck

University of Florida, Gainesville, U.S.A.

D. Acosta, P. Avery, P. Bortignon, D. Bourilkov, A. Brinkerhoff, L. Cadamuro, A. Carnes, M. Carver, D. Curry, R.D. Field, S.V. Gleyzer, B.M. Joshi, J. Konigsberg, A. Korytov, P. Ma, K. Matchev, H. Mei, G. Mitselmakher, K. Shi, D. Sperka, J. Wang, S. Wang 
Florida International University, Miami, U.S.A.

Y.R. Joshi, S. Linn

Florida State University, Tallahassee, U.S.A.

A. Ackert, T. Adams, A. Askew, S. Hagopian, V. Hagopian, K.F. Johnson, T. Kolberg, G. Martinez, T. Perry, H. Prosper, A. Saha, A. Santra, V. Sharma, R. Yohay

Florida Institute of Technology, Melbourne, U.S.A.

M.M. Baarmand, V. Bhopatkar, S. Colafranceschi, M. Hohlmann, D. Noonan, M. Rahmani, T. Roy, F. Yumiceva

University of Illinois at Chicago (UIC), Chicago, U.S.A.

M.R. Adams, L. Apanasevich, D. Berry, R.R. Betts, R. Cavanaugh, X. Chen, S. Dittmer, O. Evdokimov, C.E. Gerber, D.A. Hangal, D.J. Hofman, K. Jung, J. Kamin, C. Mills, I.D. Sandoval Gonzalez, M.B. Tonjes, N. Varelas, H. Wang, Z. Wu, J. Zhang

The University of Iowa, Iowa City, U.S.A.

M. Alhusseini, B. Bilki ${ }^{65}$, W. Clarida, K. Dilsiz ${ }^{66}$, S. Durgut, R.P. Gandrajula, M. Haytmyradov, V. Khristenko, J.-P. Merlo, A. Mestvirishvili, A. Moeller, J. Nachtman, H. Ogul ${ }^{67}$, Y. Onel, F. Ozok ${ }^{68}$, A. Penzo, C. Snyder, E. Tiras, J. Wetzel

Johns Hopkins University, Baltimore, U.S.A.

B. Blumenfeld, A. Cocoros, N. Eminizer, D. Fehling, L. Feng, A.V. Gritsan, W.T. Hung, P. Maksimovic, J. Roskes, U. Sarica, M. Swartz, M. Xiao, C. You

The University of Kansas, Lawrence, U.S.A.

A. Al-bataineh, P. Baringer, A. Bean, S. Boren, J. Bowen, J. Castle, S. Khalil, A. Kropivnitskaya, D. Majumder, W. Mcbrayer, M. Murray, C. Rogan, S. Sanders, E. Schmitz, J.D. Tapia Takaki, Q. Wang

Kansas State University, Manhattan, U.S.A.

A. Ivanov, K. Kaadze, D. Kim, Y. Maravin, D.R. Mendis, T. Mitchell, A. Modak, A. Mohammadi, L.K. Saini, N. Skhirtladze

Lawrence Livermore National Laboratory, Livermore, U.S.A.

F. Rebassoo, D. Wright

University of Maryland, College Park, U.S.A.

A. Baden, O. Baron, A. Belloni, S.C. Eno, Y. Feng, C. Ferraioli, N.J. Hadley, S. Jabeen, G.Y. Jeng, R.G. Kellogg, J. Kunkle, A.C. Mignerey, F. Ricci-Tam, Y.H. Shin, A. Skuja, S.C. Tonwar, K. Wong

Massachusetts Institute of Technology, Cambridge, U.S.A.

D. Abercrombie, B. Allen, V. Azzolini, R. Barbieri, A. Baty, G. Bauer, R. Bi, S. Brandt, W. Busza, I.A. Cali, M. D'Alfonso, Z. Demiragli, G. Gomez Ceballos, M. Goncharov, P. Harris, D. Hsu, M. Hu, Y. Iiyama, G.M. Innocenti, M. Klute, D. Kovalskyi, Y.-J. Lee, A. Levin, P.D. Luckey, B. Maier, A.C. Marini, C. Mcginn, C. Mironov, S. Narayanan, X. Niu, C. Paus, C. Roland, G. Roland, G.S.F. Stephans, K. Sumorok, K. Tatar, D. Velicanu, J. Wang, T.W. Wang, B. Wyslouch, S. Zhaozhong 
University of Minnesota, Minneapolis, U.S.A.

A.C. Benvenuti, R.M. Chatterjee, A. Evans, P. Hansen, S. Kalafut, Y. Kubota, Z. Lesko, J. Mans, S. Nourbakhsh, N. Ruckstuhl, R. Rusack, J. Turkewitz, M.A. Wadud

University of Mississippi, Oxford, U.S.A.

J.G. Acosta, S. Oliveros

University of Nebraska-Lincoln, Lincoln, U.S.A.

E. Avdeeva, K. Bloom, D.R. Claes, C. Fangmeier, F. Golf, R. Gonzalez Suarez, R. Kamalieddin, I. Kravchenko, J. Monroy, J.E. Siado, G.R. Snow, B. Stieger

State University of New York at Buffalo, Buffalo, U.S.A.

A. Godshalk, C. Harrington, I. Iashvili, A. Kharchilava, D. Nguyen, A. Parker, S. Rappoccio, B. Roozbahani

Northeastern University, Boston, U.S.A.

E. Barberis, C. Freer, A. Hortiangtham, D.M. Morse, T. Orimoto, R. Teixeira De Lima,

T. Wamorkar, B. Wang, A. Wisecarver, D. Wood

Northwestern University, Evanston, U.S.A.

S. Bhattacharya, O. Charaf, K.A. Hahn, N. Mucia, N. Odell, M.H. Schmitt, K. Sung, M. Trovato, M. Velasco

University of Notre Dame, Notre Dame, U.S.A.

R. Bucci, N. Dev, M. Hildreth, K. Hurtado Anampa, C. Jessop, D.J. Karmgard, N. Kellams,

K. Lannon, W. Li, N. Loukas, N. Marinelli, F. Meng, C. Mueller, Y. Musienko ${ }^{32}$, M. Planer,

A. Reinsvold, R. Ruchti, P. Siddireddy, G. Smith, S. Taroni, M. Wayne, A. Wightman,

M. Wolf, A. Woodard

The Ohio State University, Columbus, U.S.A.

J. Alimena, L. Antonelli, B. Bylsma, L.S. Durkin, S. Flowers, B. Francis, A. Hart, C. Hill, W. Ji, T.Y. Ling, W. Luo, B.L. Winer, H.W. Wulsin

Princeton University, Princeton, U.S.A.

S. Cooperstein, P. Elmer, J. Hardenbrook, P. Hebda, S. Higginbotham, A. Kalogeropoulos,

D. Lange, M.T. Lucchini, J. Luo, D. Marlow, K. Mei, I. Ojalvo, J. Olsen, C. Palmer,

P. Piroué, J. Salfeld-Nebgen, D. Stickland, C. Tully

University of Puerto Rico, Mayaguez, U.S.A.

S. Malik, S. Norberg

Purdue University, West Lafayette, U.S.A.

A. Barker, V.E. Barnes, S. Das, L. Gutay, M. Jones, A.W. Jung, A. Khatiwada, D.H. Miller,

N. Neumeister, C.C. Peng, H. Qiu, J.F. Schulte, J. Sun, F. Wang, R. Xiao, W. Xie

Purdue University Northwest, Hammond, U.S.A.

T. Cheng, J. Dolen, N. Parashar 
Rice University, Houston, U.S.A.

Z. Chen, K.M. Ecklund, S. Freed, F.J.M. Geurts, M. Guilbaud, M. Kilpatrick, W. Li, B. Michlin, B.P. Padley, J. Roberts, J. Rorie, W. Shi, Z. Tu, J. Zabel, A. Zhang

University of Rochester, Rochester, U.S.A.

A. Bodek, P. de Barbaro, R. Demina, Y.t. Duh, J.L. Dulemba, C. Fallon, T. Ferbel, M. Galanti, A. Garcia-Bellido, J. Han, O. Hindrichs, A. Khukhunaishvili, K.H. Lo, P. Tan, R. Taus, M. Verzetti

Rutgers, The State University of New Jersey, Piscataway, U.S.A.

A. Agapitos, J.P. Chou, Y. Gershtein, T.A. Gómez Espinosa, E. Halkiadakis, M. Heindl, E. Hughes, S. Kaplan, R. Kunnawalkam Elayavalli, S. Kyriacou, A. Lath, R. Montalvo, K. Nash, M. Osherson, H. Saka, S. Salur, S. Schnetzer, D. Sheffield, S. Somalwar, R. Stone, S. Thomas, P. Thomassen, M. Walker

University of Tennessee, Knoxville, U.S.A.

A.G. Delannoy, J. Heideman, G. Riley, K. Rose, S. Spanier, K. Thapa

Texas A\&M University, College Station, U.S.A.

O. Bouhali ${ }^{69}$, A. Castaneda Hernandez ${ }^{69}$, A. Celik, M. Dalchenko, M. De Mattia, A. Delgado, S. Dildick, R. Eusebi, J. Gilmore, T. Huang, T. Kamon ${ }^{70}$, S. Luo, R. Mueller, Y. Pakhotin, R. Patel, A. Perloff, L. Perniè, D. Rathjens, A. Safonov, A. Tatarinov

Texas Tech University, Lubbock, U.S.A.

N. Akchurin, J. Damgov, F. De Guio, P.R. Dudero, S. Kunori, K. Lamichhane, S.W. Lee, T. Mengke, S. Muthumuni, T. Peltola, S. Undleeb, I. Volobouev, Z. Wang

Vanderbilt University, Nashville, U.S.A.

S. Greene, A. Gurrola, R. Janjam, W. Johns, C. Maguire, A. Melo, H. Ni, K. Padeken, J.D. Ruiz Alvarez, P. Sheldon, S. Tuo, J. Velkovska, Q. Xu

\section{University of Virginia, Charlottesville, U.S.A.}

M.W. Arenton, P. Barria, B. Cox, R. Hirosky, M. Joyce, A. Ledovskoy, H. Li, C. Neu, T. Sinthuprasith, Y. Wang, E. Wolfe, F. Xia

\section{Wayne State University, Detroit, U.S.A.}

R. Harr, P.E. Karchin, N. Poudyal, J. Sturdy, P. Thapa, S. Zaleski

University of Wisconsin - Madison, Madison, WI, U.S.A.

M. Brodski, J. Buchanan, C. Caillol, D. Carlsmith, S. Dasu, L. Dodd, S. Duric, B. Gomber,

M. Grothe, M. Herndon, A. Hervé, U. Hussain, P. Klabbers, A. Lanaro, A. Levine, K. Long,

R. Loveless, T. Ruggles, A. Savin, N. Smith, W.H. Smith, N. Woods

$\dagger$ : Deceased

1: Also at Vienna University of Technology, Vienna, Austria

2: Also at IRFU, CEA, Université Paris-Saclay, Gif-sur-Yvette, France

3: Also at Universidade Estadual de Campinas, Campinas, Brazil

4: Also at Federal University of Rio Grande do Sul, Porto Alegre, Brazil 
5: Also at Université Libre de Bruxelles, Bruxelles, Belgium

6: Also at Institute for Theoretical and Experimental Physics, Moscow, Russia

7: Also at Joint Institute for Nuclear Research, Dubna, Russia

8: Now at British University in Egypt, Cairo, Egypt

9: Also at Zewail City of Science and Technology, Zewail, Egypt

10: Now at Helwan University, Cairo, Egypt

11: Also at Department of Physics, King Abdulaziz University, Jeddah, Saudi Arabia

12: Also at Université de Haute Alsace, Mulhouse, France

13: Also at Skobeltsyn Institute of Nuclear Physics, Lomonosov Moscow State University, Moscow, Russia

14: Also at CERN, European Organization for Nuclear Research, Geneva, Switzerland

15: Also at RWTH Aachen University, III. Physikalisches Institut A, Aachen, Germany

16: Also at University of Hamburg, Hamburg, Germany

17: Also at Brandenburg University of Technology, Cottbus, Germany

18: Also at Institute of Nuclear Research ATOMKI, Debrecen, Hungary

19: Also at MTA-ELTE Lendület CMS Particle and Nuclear Physics Group, Eötvös Loránd University, Budapest, Hungary

20: Also at Institute of Physics, University of Debrecen, Debrecen, Hungary

21: Also at Indian Institute of Technology Bhubaneswar, Bhubaneswar, India

22: Also at Institute of Physics, Bhubaneswar, India

23: Also at Shoolini University, Solan, India

24: Also at University of Visva-Bharati, Santiniketan, India

25: Also at Isfahan University of Technology, Isfahan, Iran

26: Also at Plasma Physics Research Center, Science and Research Branch, Islamic Azad University, Tehran, Iran

27: Also at Università degli Studi di Siena, Siena, Italy

28: Also at International Islamic University of Malaysia, Kuala Lumpur, Malaysia

29: Also at Malaysian Nuclear Agency, MOSTI, Kajang, Malaysia

30: Also at Consejo Nacional de Ciencia y Tecnología, Mexico city, Mexico

31: Also at Warsaw University of Technology, Institute of Electronic Systems, Warsaw, Poland

32: Also at Institute for Nuclear Research, Moscow, Russia

33: Now at National Research Nuclear University 'Moscow Engineering Physics Institute' (MEPhI), Moscow, Russia

34: Also at St. Petersburg State Polytechnical University, St. Petersburg, Russia

35: Also at University of Florida, Gainesville, U.S.A.

36: Also at P.N. Lebedev Physical Institute, Moscow, Russia

37: Also at California Institute of Technology, Pasadena, U.S.A.

38: Also at Budker Institute of Nuclear Physics, Novosibirsk, Russia

39: Also at Faculty of Physics, University of Belgrade, Belgrade, Serbia

40: Also at INFN Sezione di Pavia ${ }^{a}$, Università di Pavia ${ }^{b}$, Pavia, Italy

41: Also at University of Belgrade, Faculty of Physics and Vinca Institute of Nuclear Sciences, Belgrade, Serbia

42: Also at Scuola Normale e Sezione dell'INFN, Pisa, Italy

43: Also at National and Kapodistrian University of Athens, Athens, Greece

44: Also at Riga Technical University, Riga, Latvia

45: Also at Universität Zürich, Zurich, Switzerland

46: Also at Stefan Meyer Institute for Subatomic Physics (SMI), Vienna, Austria

47: Also at Adiyaman University, Adiyaman, Turkey 
48: Also at Istanbul Aydin University, Istanbul, Turkey

49: Also at Mersin University, Mersin, Turkey

50: Also at Piri Reis University, Istanbul, Turkey

51: Also at Gaziosmanpasa University, Tokat, Turkey

52: Also at Ozyegin University, Istanbul, Turkey

53: Also at Izmir Institute of Technology, Izmir, Turkey

54: Also at Marmara University, Istanbul, Turkey

55: Also at Kafkas University, Kars, Turkey

56: Also at Istanbul Bilgi University, Istanbul, Turkey

57: Also at Hacettepe University, Ankara, Turkey

58: Also at Rutherford Appleton Laboratory, Didcot, United Kingdom

59: Also at School of Physics and Astronomy, University of Southampton, Southampton, United Kingdom

60: Also at Monash University, Faculty of Science, Clayton, Australia

61: Also at Bethel University, St. Paul, U.S.A.

62: Also at Karamanoğlu Mehmetbey University, Karaman, Turkey

63: Also at Utah Valley University, Orem, U.S.A.

64: Also at Purdue University, West Lafayette, U.S.A.

65: Also at Beykent University, Istanbul, Turkey

66: Also at Bingol University, Bingol, Turkey

67: Also at Sinop University, Sinop, Turkey

68: Also at Mimar Sinan University, Istanbul, Istanbul, Turkey

69: Also at Texas A\&M University at Qatar, Doha, Qatar

70: Also at Kyungpook National University, Daegu, Korea 